\title{
WHEN IS A COMPLEX FIBERED BY A SUBCOMPLEX?
}

\author{
BY \\ S. Y. HUSSEINI(1)
}

Introduction. Suppose that $(B, F)$ is a pair of spaces of the homotopy type of countable CW-complexes. Problem 10 of Massey's list of 1955 [6] poses the question of when $(B, F)$ is homotopically equivalent (i.e., there is a homotopy commutative diagram

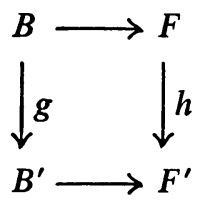

such that $g$ and $h$ are homotopy equivalences) to a pair $\left(B^{\prime}, F^{\prime}\right)$ such that $B^{\prime}$ is fibered, in the sense of Serre, by $F^{\prime}$ with base-space $Y$, for some suitable $Y$. If $B^{\prime}$ is contractible and $F^{\prime}$ fibres $B^{\prime}$, then $F^{\prime}$ (and hence $F$ ) is of the homotopy type of the loopspace $\Omega(Y)$ (see e.g. [9]). Conversely, if $B$ is contractible and if $F$ is of the homotopy type of a loopspace $\Omega(Y)$, then there is a pair $\left(B^{\prime}, F^{\prime}\right)$ homotopically equivalent to $(B, F)$ such that $F^{\prime}$ fibres $B^{\prime}$ with base-space $Y$ (see [1], [11]). In both cases, $Y$ is of homotopy type of the projective space of $F$, which is inductively defined by means of Hopf fibrations. Since projective spaces and Hopf fibrations have proved useful in the study of $H$-spaces, I have tried to preserve these features in the solution of the general problem which I offer in this paper.

The general idea of the solution is to consider first $\Omega(B, F)$, the space of paths beginning in some fixed point $b^{0}$ and ending in $F$, as a right $\Omega(B)$-space, and then to investigate certain multiplications on $\Omega(B, F)$ which extend this action. These multiplications yield fibrations of the Hopf type, in which the total spaces are the manifold joins of $F$ with itself when $F$ is a suspension and are "twisted" manifold joins of $F$ in general (see $\S 6$ ). One also gets spaces which resemble the classical projective spaces; in particular, one gets a filtration of $Y$ (or rather a space of the homotopy type of $Y$ ) by subspaces determined by $F$ and $B$ (see $\S 6$ ).

The solution is given in the context of constructions of the reduced product type (RPT-constructions for short). To be able to handle the right action of $\Omega(B)$ on $\Omega(B, F)$, one needs a relative theory of RPT-constructions. This can easily be

Received by the editors September 20, 1965.

(1) While writing this paper the author was partially supported by NSF Contract GP-3857. 
developed as follows. (All complexes are supposed to be special, i.e., countable CW-complexes with a single 0 -cell.) Suppose $A$ is an $H$-complex which is a limit of RPT-complexes [3]. Then $A$, like RPT-complexes, is essentially an associative $H$-complex with a nondegenerate multiplication in the sense that the product of two cells of $A$ is again a cell of $A$. The domain of the theory is the category whose objects are complexes containing $A$ as a subcomplex and on which $A$ acts on both sides nondegenerately in the sense given above. (The objects are called two-sided A-complexes.) The maps of the category are cellular maps which respect the action of $A$. (The maps are called two-sided invariant maps.) Having established the category, one proceeds in a manner similar to that of the absolute theory as developed in [3]. Thus if $X$ is a two sided $A$-complex, then a set of A-relations in $k$ variables on $X$,

$$
\mathscr{M}_{k}(X)=\left(M_{0}(X), M_{1}(X), \cdots, M_{k}(X) ; \mu_{p, q}, A\right),
$$

is given by a two-sided $A$-complex $M_{k}(X)$ filtered by two-sided $A$-subcomplexes $M_{p}(X)$, such that $M_{0}(X)=A$ and $M_{1}(X)=X$, and by two-sided invariant maps $\mu_{p, q}$ which are associative, with a two-sided identity, and take $M_{p}(X) \times M_{q}(X)$ into $M_{p+q}(X)$ nondegenerately. (If $A$ consists of a single 0 -cell only, then this notion of a set of relations is that given in [3] for the absolute case.) One also shows, as in the absolute theory, that $X$ generates a universal $H$-complex modulo any $\mathscr{M}_{k}(X)$. The details of the theory are given in the first and third sections.

The application of the relative theory of RPT-constructions to the problem mentioned at the beginning of this introduction is a continuation of the reformulation of Stasheff's $A_{n}$-structures given in [3]. First, replace $\Omega(B)$ by a complex $A$ which is a limit of RPT-complexes and $\Omega(B, F)$ by a right $A$-complex $U$. It turns out that the existence of a fibration

$$
F^{\prime} \rightarrow B^{\prime} \rightarrow Y
$$

such that $\left(B^{\prime}, F^{\prime}\right)$ is equivalent to the given pair $(B, F)$ implies, first, that $U \sim \Omega(B, F)$ is of the homotopy type of a two-sided $A$-complex $X$ and, second, that $X$ admits a set of $A$-relations for all $n \geqq 1$

$$
\mathscr{M}_{n}(X)=\left(M_{0}(X), M_{1}(X), \cdots, M_{n}(X) ; \mu_{p, q}, A\right)
$$

such that $M_{i}$ is a deformation retract of $M_{i+1}$, where $1 \leqq i \leqq n-1$, the deformation being given by two-sided invariant maps. ( $X$ is said to admit a $Q_{n}$-structure if it admits a set of $A$-relations such as $\mathscr{M}_{n}(X)$; if $A$ consists of one 0-cell only, then $Q_{n}$-and $A_{n}$-structures agree (see [3]). Conversely, the existence of a two-sided $A$-complex $X$ of the homotopy type of $U \sim \Omega(B, F)$ and of a $Q_{n}$-structure on $X$ imply together the existence of a fibration,

$$
F_{n} \rightarrow B^{\prime} \rightarrow Y_{n},
$$


such that the pair $\left(B^{\prime}, F_{n}\right)$ is equivalent to the pair $(B, F)$ in a range that increases with $n$ (see $\S 5$ ). This reduces Massey's problem 10 to a problem on the existence of $Q_{n}$-structures.

To get the fibrations of the Hopf type from the set of relations defining a $Q_{n}$-structure on $X \sim U \sim \Omega(B, F)$ demands a point of view quite different from the one usually employed. This point of view can be roughly described as follows. Suppose $G$ is an associative $H$-space and consider the $k$ th stage Hopf-fibration of $G$,

$$
G \stackrel{i_{k}}{\longrightarrow} E_{k} \stackrel{p_{k}}{\longrightarrow} P_{k},
$$

where $E_{k}$ is the $(k+1)$-fold join of $G$ with itself and $P_{k}=E_{k} / G$, the so-called projective space of $G$ (see [1], [11]). Then $p_{k}$ induces a fibration

$$
\Omega P_{k} \longrightarrow \tilde{G} \stackrel{\tilde{l}_{k}}{\longrightarrow} E_{k},
$$

from the path-space fibration over $P_{k}$,

$$
\Omega P_{k} \longrightarrow P\left(P_{k}\right) \longrightarrow P_{k} \text {. }
$$

Note that $\tilde{G}$ is an $\Omega\left(P_{k}\right)$-space of the homotopy type of $G, \tilde{i}_{k}$ equivalent to $i_{k}$, and $p_{k}$ the classifying map of the fibration (II). In Massey's problem, $M_{1}(X)=X$ is analagous to $G$, and ${ }^{k} M$, to $\Omega P_{k}$ where ${ }^{k} M$ is the RPT-complex generated by $X$ modulo $\mathscr{M}_{k}(X)$. What I do here is construct (II) first and then obtain (I) from it by constructing the classifying map $p_{k}$. To construct (II) one must replace $M_{1}$ by a homotopically equivalent right ${ }^{k} M$-complex $N_{k}$ and then show how in general a right $A$-complex $N$ is quasi-fibered by $A$ over a base-complex $N / / A$ similar to an orbit space. The latter problem is answered by Theorem (2.2) of $\S 2-$ a result of independent interest which plays a primary role in this work. The complex $N_{k}$ is constructed in $\S 8$. Now, Theorem (2.2) shows that $N_{k}$ is quasi-fibered by $A$ over the orbit complex $N_{k} / / A$. The complex $N_{k} / / A$ is shown to be of the homotopy type of the $(k+1)$-fold join of $F$ with itself, when $F$ is a suspension, and is the "relative" ( $k+1)$-fold join of $M_{1} / / A$ in general (see $\S \S 6$ and 8). The quasifibration of any right $A$-complex $N$ over $N / / A$ is shown, in $\S 4$, to admit a classifying map. One obtains (I) from (II) by taking $p_{k}$ to be the classifying maps of (II).

I would like to express my thanks to the referee of this paper for his suggestions which contributed to its better organization and for the title, a more descriptive one than the original.

1. Preliminary notions and definitions. Suppose $A$ is a special complex in the sense of James, i.e., a countable CW-complex with a single 0-cell $a^{0}$. Take $a^{0}$ to be the basepoint of $A$. Assume that $A$ is an $H$-complex with multiplication $\alpha$. (Recall that, by definition, $\alpha$ is cellular, associative, and has $a^{0}$ as an identity.) Suppose also that $A$ is filtered by subcomplexes

$$
A_{0}=\left\{a^{0}\right\} \subset A_{1} \subset \cdots \subset A_{p} \subset \cdots
$$


and $\alpha$ takes $A_{p} \times A_{q}$ into $A_{p+q}$ for all $p$ and $q$. Write $A_{n}(X)$ for $A_{n}$, where $A_{1}=X$. Then we say that $A$ is a complex of the reduced product type (an RPT-complex for short) if, and only if, the set

$$
\mathscr{A}_{k}(X)=\left(A_{0}(X), A_{1}(X), \cdots, A_{k}(X) ; \alpha_{p, q}\right),
$$

where $\alpha_{p, q}=\alpha \mid A_{p}(X) \times A_{q}(X)$, is a set of relations in the sense of [3], for all $k \geqq 2$. If, moreover, there is an integer $m$ such that

$$
A_{p}(X)=\bigcup_{r+s=p, r, s>0} \alpha_{r, s}\left(A_{r}(X) \times A_{s}(X)\right),
$$

for all $p>m$, then we say that $A$ is of $r a n k \leqq m$. Otherwise we say $A$ is of infinite rank. (This notion of an RPT-complex is slightly different from that given in [3]. An RPT-complex in the sense of [3] is always of finite rank. Here we are going to admit RPT-complexes of arbitrary rank.)

Suppose that $B$ is a special complex containing $A$ as a subcomplex and that it filtered by an ascending sequence of subcomplexes

$$
B_{0}=A \subset \cdots \subset B_{m} \subset \cdots .
$$

Assume that there is a cellular mapping

$$
\eta: B \times A \rightarrow B,
$$

with the following properties:

(1.1) $)_{\mathrm{a}}$ the basepoint $a^{0}$ of $A$ is the identity, i.e. $\eta\left(b, a^{0}\right)=b$, for all $b$ in $B$;

$(1.1)_{\mathrm{b}} \quad \eta$ is associative, i.e. $\eta(\eta \times 1)=\eta(1 \times \eta)$; and

(1.1) $\quad \eta$ restricted to $A \times A$ is just $\alpha$, the multiplication in $A$.

So far, what we have is a complex $B$ and an action on the right by $A$ on $B$. However, one must restrict one's attention, in the context of reduced products, to those actions which are nondegenerate in the sense that the product of a cell in $B$ by a cell in $A$ is a cell in $B$. The precise condition is the following:

$\eta$ takes $B_{m} \times A_{n}$ to $B_{m+n}$ and imbeds the complex $\left[B_{m}, A_{n}\right]$ obtained by (1.1) $)_{\mathrm{d}} \quad$ attaching $B_{m} \times A_{n}$ to $B_{m+n-1}$ by the map $\eta \mid B_{m-1} \times A_{n} \cup B_{m} \times A_{n-1}$, into $B_{m+n}$ as a subcomplex.

Definition (1.2). Suppose $B$ is a special complex and $A$ an RPT-complex imbedded in $B$ as a subcomplex. $B$ is said to be a right $A$-complex if it is filtered by an ascending sequence

$$
B_{0}=A \subset \cdots \subset B_{m} \subset \cdots
$$

and there is a cellular mapping

$$
\eta: B \times A \rightarrow B
$$

subject to the conditions $(1.1)_{\mathrm{a}}, \cdots,(1.1)_{\mathrm{d}}$ given above. 
Similarly, we define a left $A$-complex $B$ to be a filtered special complex with a mapping

$$
\phi: A \times B \rightarrow B
$$

subject to conditions similar to $(1.1)_{\mathrm{a}}, \cdots,(1.1)_{\mathrm{d}}$ given above. $A$ two-sided $A$-complex $B$ is, by definition, a left and right $A$-complex such that the left and right actions of $A$ on $B$ commute with each other.

In studying $A$-complexes one can vary the given filtration to a certain extent (see $\S 3$ below). In fact, one can always replace an arbitrary filtration by the one given by its skeletons, as can be easily checked. Note also that nondegeneracy is defined by means of the filtration, rather than by requiring that the product on any two cells be also a cell, because quite often one gets naturally defined filtrations (see §5), with which it is more natural to work. Note also that the filtrations of the left and right actions a two-sided complex need not be the same.

An example of a two-sided $A$-complex is given by taking $B=A$ and letting $\eta$ and $\phi$ be the multiplication $\alpha$ in $A$. Other examples are obtained by taking first a special complex $C$ containing $A$ as a subcomplex, and then setting

$$
B=A \cup C \times A,
$$

where the complex $C \times A$ is attached to $A$ by the multuiplication $\alpha$ of $A$. Clearly $B$ is a right $A$-complex with $A$ acting naturally on the right. Similarly, one can define $A \cup A \times C$ and $A \cup A \times C \times A$. The former is a left $A$-complex, and the latter is a two-sided $A$-complex. In fact, any $A$-complex is filtered by subcomplexes of one of these three types. To see this, suppose $A$ is an RPT-complex of arbitrary rank, and let $B$ be a right $A$-complex. By definition, let $\widetilde{B}_{1}$ be the smallest subcomplex of $B$ which contains $A$ and the cells of $B-A$ of lowest dimension. Then the complex

$$
B=A \cup \widetilde{B}_{1} \times A,
$$

where $\widetilde{B}_{1} \times A$ is attached to $A$ by the multiplication $A \times A \rightarrow A$, is a subcomplex of $B$, as follows easily from (1.1) $)_{\mathrm{d}}$. Suppose we have been able to define a sequence of subcomplexes of $B$,

$$
\widetilde{B}_{0}=A \subset \widetilde{B}_{1} \subset \cdots \subset \widetilde{B}_{m},
$$

and a sequence of right $A$-subcomplexes of $B$,

such that

$$
B_{0}=A \subset B_{1} \subset \cdots \subset B_{m},
$$

(i) $\widetilde{B}_{i} \supset B_{i-1}$ and

(ii) $B_{i}=B_{i-1} \cup \widetilde{B}_{i} \times A$,

where the attaching map is given by the action of $B_{i-1} \times A \rightarrow B_{i-1}$. If $B_{m} \neq B$, define $\widetilde{B}_{m+1}$ to be the smallest subcomplex of $B$ containing $B_{m}$ and the cells of $B-B_{m}$ of smallest dimension. Now, if we let 


$$
B_{m+1}=B_{m} \cup B_{m+1} \times A,
$$

where the attaching map is given by the action of $B_{m} \times A \rightarrow B_{m}$, we get what we wish. Hence we have the following proposition.

Proposition (1.3). Suppose $B$ is a right A-complex, where $A$ is an RPTcomplex of arbitrary rank. Then there is a sequence of subcomplexes of $B$,

$$
A=\widetilde{B}_{0} \subset \widetilde{B}_{1} \subset \cdots \subset \widetilde{B}_{m} \subset \cdots,
$$

and a sequence of right A-subcomplexes of $B$,

such that

$$
A=B_{0} \subset B_{1} \subset \cdots \subset B_{m} \subset \cdots,
$$

(i) $B_{m-1} \subset \widetilde{B}_{m}$;

(ii) $B_{m}=B_{m-1} \cup \widetilde{B}_{m} \times A, m \geqq 1$

(the attaching map being the action $B_{m-1} \times A \rightarrow B_{m-1}$ ); and

(iii) $B=\lim _{m} B_{m}$.

Similarly, one can prove the following proposition.

Proposition (1.4). Suppose B is a two-sided A complex, where $A$ is an RPTcomplex of arbitrary rank. Then there is an ascending sequence of subcomplexes of $B$,

$$
A=\widetilde{B}_{0} \subset \widetilde{B}_{1} \subset \cdots \subset \widetilde{B}_{m} \subset \cdots,
$$

and an ascending sequence of two sided A-subcomplexes of $B$,

such that

$$
A=B_{0} \subset B_{1} \subset \cdots \subset B_{m} \subset \cdots,
$$

(i) $B_{m-1} \subset \widetilde{B}_{m}$;

(ii) $B_{m}=B_{m-1} \cup A \times \widetilde{B}_{m} \times A, m \geqq 1$

(the attaching map being the action $A \times B_{m-1} \times A \rightarrow B_{m-1}$ ); and

(iii) $B=\lim _{m} B_{m}$.

There is a similar representation for the left $A$-complexes. Note that these representations depend on the cellular decomposition of $B$ and not on the filtrations.

Suppose $B$ is a right $A$-complex and $B^{\prime}$ a right $A^{\prime}$-complex. By definition, a right invariant map

$$
f: B \rightarrow B^{\prime}
$$

is a cellular map of $B$ into $B^{\prime}$ such that

$$
f \phi(x, a)=\phi^{\prime}(f(x), f(y)),
$$

where $\phi$ and $\phi^{\prime}$ are the actions of $A$ and $A^{\prime}$ on $B$ and $B^{\prime}$, for all $x$ in $B$ and $a$ in $A$, and $f \mid A$ is a homomorphism of $A$ into $A^{\prime}$. Similarly we define a left invariant 
map, and a two-sided invariant map. A right (left, two-sided) $A$-map $f$ is a right (left, two-sided) invariant map such that $A=A^{\prime}$ and $f \mid A=1$.

Suppose next that $B$ and $B^{\prime}$ are, respectively, right and left $A$-complexes, and denote the actions of $A$ by $\phi$ and $\eta^{\prime}$. We wish to join $B$ to $B$ ' along the "orbits" of $A$. Consider, therefore, the special complex

$$
B \times A \times B^{\prime} .
$$

Suppose $x_{1}=\left(b_{1}, a_{1}, b_{1}^{\prime}\right)$ and $x_{2}=\left(b_{2}, a_{2}, b_{2}^{\prime}\right)$ are two elements of $B \times A \times B^{\prime}$. Then we shall say that $x_{1}$ is an amalgam of $x_{2}$ if, and only if,

$$
\begin{aligned}
& b_{1}=\phi\left(b_{2}, a_{2}\right), a_{1}=a^{0}, \text { and } b_{1}^{\prime}=b_{2}^{\prime}, \text { or } \\
& b_{1}^{\prime}=\eta^{\prime}\left(a_{2}, b_{2}^{\prime}\right), a_{1}=a^{0}, \text { and } b_{1}=b_{2},
\end{aligned}
$$

where $a_{1}, a_{2}$ are in $A, b_{1}, b_{2}$ in $B$, and $b_{1}^{\prime}, b_{2}^{\prime}$ in $B^{\prime}$, and $a^{0}$ is the basepoint of $A$. This notion of amalgamation is similar to that of [3] and generates in a similar manner an equivalence relation. Let $B \times{ }_{A} B^{\prime}$ be the quotient space of $B \times A \times B^{\prime}$ modulo this equivalence relation, and give $B \times{ }_{A} B^{\prime}$ the quotient topology. Clearly $B \times{ }_{A} B^{\prime}$ is a covariant functor in two variables. The following result can be proved in the same manner as Theorem (2.4) of [3].

Theorem (1.4). Suppose $B$ and $B^{\prime}$ are right and left $A$-complexes. Then the cellular structure of $B \times A \times B^{\prime}$ induces the structure of a special complex on $B \times{ }_{A} B^{\prime}$. Also, if $B$ and $B^{\prime}$ are two-sided $A$-complexes, then the left action of $A$ on $B$ and the right action of $A$ on $B^{\prime}$ make $B \times{ }_{A} B^{\prime}$ a two-sided $A$-complex.

By definition, $B \times{ }_{A} B^{\prime}$ is the $A$-cartesian product of $B$ and $B^{\prime}$. Observe that the imbeddings of $B$ and $B^{\prime}$ as the first and last factor in $B \times A \times B^{\prime}$ induce imbeddings of $B$ and $B^{\prime}$ in $B \times{ }_{A} B^{\prime}$, as subcomplexes whose intersection is $A$. Note also that if $A=\left\{a^{0}\right\}$, a single 0 -cell, then an $A$-complex is just a special complex, and $B \times{ }_{A} B^{\prime}$ is the usual cartesian product.

2. The quasi-fibration of an $A$-complex over its orbit space. Recall that a map

$$
p: E \rightarrow B
$$

of the space $E$ onto the space $B$ is a quasi-fiber map if, and only if,

$$
p_{*}: \pi_{i}\left(E, p^{-1}(x), y\right)=\pi_{i}(B, x),
$$

for all $x$ in $B, y$ in $p^{-1}(x)$, and $i \geqq 0$ [2]. Also recall that if $A$ is an associative $H$-space, then a left principal quasi-fiber map for $A$ is a quasi-fiber map

$$
p: E \rightarrow B,
$$

together with a left action of $A$ on $E$, 


$$
\eta: A \times E \rightarrow E,
$$

such that

(i) $\eta\left(a^{0}, e\right)=e$,

where $a^{0}$ is the 0-cell of $A$ and $e$ is in $E$;

(ii) $\eta(A \times e) \subset p^{-1}(p(e))$,

$p^{-1}(p(e))$ being the fiber through $e$; and

(iii) the mapping

$$
\eta_{e}: A \rightarrow p^{-1}(p(e))
$$

which takes $a$ to $\eta(a, e)$ is a weak homotopy equivalence [1]. Similarly one defines a right principal quasi-fibration.

The object of this section is to define the complex of orbits of $A$ in $N$ and to show that the natural projection of $N$ on its orbit complex is a quasi-fibration.

Suppose $A$ is an RPT-complex of arbitrary rank, and let $N$ be a right $A$-complex as in $\S 1$. Denote the action of $A$ on $N$ by $\eta$. Two elements $n$ and $n^{\prime}$ of $N$ are said to be equivalent if, and only if, there is a chain of elements $n_{1}, \cdots, n_{r}$ in $N$ such that, for every consecutive pair $n_{s}, n_{s+1}$, there is an element $a$ in $A$ such that either $\eta\left(n_{s}, a\right)=n_{s+1}$ or $\eta\left(n_{s+1}, a\right)=n_{s}$. Denote the quotient space by $N / / A$, and give it the quotient topology. $N / / A$ is, by definition, the orbit complex of $\eta$. Suppose $p$ is the natural projection of $N$ onto $N / / A$. Now, according to Proposition (1.3) of $\S 1$, there is an ascending sequence

$$
N_{0} \subset \cdots \subset N_{m} \subset \cdots
$$

of right $A$-subcomplexes of $N$ such that

$$
N_{0}=A, \text { and } N_{m}=N_{m-1} \cup \tilde{N}_{m} \times A, \quad m>0,
$$

where $\tilde{N}_{m}$ is the smallest subcomplex of $N$ containing $N_{m-1}$ and the cells of $N-N_{m-1}$ of lowest dimension. We conclude from this that the cellular structure of $N$ induces the structure of a special complex on $N / / A$ and that the natural map,

$$
p: N \rightarrow N / / A,
$$

is cellular.

TheORem (2.2). Suppose $N$ is a right A-complex. Then the natural projection

$$
p: N \rightarrow N / / A
$$

is a principal quasi-fibration.

Theorem (2.2) is a special case of the following theorem.

TheOREM (2.2'). Suppose $N$ and $N^{\prime}$ are right and left A-complexes. Then 
(i) the map

$$
p^{\prime}: N \times{ }_{A} N^{\prime} \rightarrow N / / A,
$$

induced by the projection on the first factor, is a quasi-fibration with fibre $N^{\prime}$; and

(ii) the map

$$
\gamma_{n}: N^{\prime} \rightarrow p^{\prime-1}(p(n)),
$$

which takes the point $n^{\prime}$ to $n \times_{A} n^{\prime}$, is a homotopy equivalence for all $n$ in $N$.

Proof. Consider the ascending sequence (2.1) given above. If $m=0$, then $N_{0} / / A=\tilde{a}^{0}$, the 0 -cell of $N / / A$, and, hence

$$
p_{0}^{\prime}: N_{0} \times_{A} N^{\prime} \rightarrow \tilde{a}^{0}, \quad p_{0}^{\prime}=p \mid N_{0}
$$

is trivially a quasi-fibration; and, since $A$ is arcwise connected, condition (ii) is also satisfied. Suppose we have proved that the map

$$
p_{m}^{\prime}: N_{m} \times_{A}: N^{\prime} \rightarrow N_{m} / / A, \quad p_{m}^{\prime}=p \mid N_{m}
$$

is a quasi-fibration with fiber $N^{\prime}$ and such that (ii) of the theorem is satisfied when $N=N_{m}$. We wish to do the same thing for the case $m+1$. Note that

$$
N_{m+1}-N_{m}=\bigcup \dot{D}^{n_{r}} \times A
$$

where $D^{n_{r}}$ ranges over the cells of $\tilde{N}_{m+1}-N_{m}$ and $\dot{D}^{k}$ denotes the interior of $D^{k}$. Hence,

$$
N_{m+1} \times_{A} N^{\prime}-N_{m} \times_{A} N^{\prime}=\bigcup \dot{D}^{n_{r}} \times N^{\prime} .
$$

Moreover, we can write

$$
N_{m+1} / / A=U \cup V
$$

where

$$
V=p_{m+1}^{\prime}\left(\bigcup D^{n_{r}}(3 / 4) \times N^{\prime}\right)
$$

( $D^{n_{r}}(3 / 4)$ being the $n_{r}$-disc of radius $\left.3 / 4\right)$, and

where

$$
U=p_{m+1}^{\prime}\left(N_{m+1}(1 / 4) \times_{A} N^{\prime}\right),
$$

$$
N_{m+1}(1 / 4)=N_{m} \cup \bigcup\left(D^{n_{r}}-\dot{D}^{n_{r}}(1 / 4)\right) \times A .
$$

Clearly $V$ is a distinguished set. Hence, to finish the proof we need to show that $U$ is also distinguished since $U \cap V$ is distinguished (see (2.2) of [2]). But, according to $(2.10)$ of [2], we need prove only that the map

$$
\gamma_{n}: N^{\prime} \rightarrow p_{m+1}^{\prime-1}\left(p_{m+1}^{\prime}(n)\right)
$$


which takes $n^{\prime}$ to $n \times{ }_{A} n^{\prime}$, for all $n$ in $N_{m+1}$ is a homotopy equivalence. (This also proves the assertion (ii) of the theorem when $N=N_{m+1}$.) Observe that

$$
p_{m}^{\prime-1}\left(p_{m}^{\prime}(n)\right)=p_{m+1}^{\prime-1}\left(p_{m+1}^{\prime}(n)\right),
$$

if $n$ is in $N_{m}$. In this case the induction assumption implies $\gamma_{n}$ is a homotopy equivalence. If $n$ is in $N_{m+1}-N_{m}$, then

$$
n=(\tilde{n}, a) \text {, }
$$

for some $\tilde{n}$ in $\tilde{N}_{m+1}-N_{m}$ and $a$ in $A$. Hence $\gamma_{n}$ takes $n^{\prime}$ to $\left(\tilde{n}, a n^{\prime}\right)$. Since $A$ is arcwise connected and

$$
p_{m+1}^{\prime-1}\left(p_{m+1}^{\prime}(n)\right)=\tilde{n} \times N^{\prime}
$$

$\gamma_{n}$ is a homotopy equivalence. This establishes the induction step and hence the theorem.

Remark (2.3). Suppose $N^{\prime}$ is a left $A$-complex. Then one can define an equivalence relation on $N^{\prime}$, as was done for right $A$-complexes. Denote the quotient space by $A \| N^{\prime}$. Then $A \| N^{\prime}$ inherits from $N^{\prime}$ a natural cellular structure, and the projection

$$
p^{\prime}: N^{\prime} \rightarrow A \backslash \mid N^{\prime}
$$

is again a principal quasi-fibration. Moreover, if $N$ is a right $A$-complex, then the projection

$$
N \times{ }_{A} N^{\prime} \rightarrow A \| N^{\prime}
$$

induced by the projection on the second factor is a quasi-fibration with fiber $N$.

3. Constructions relative to an RPT-complex. Very often one has a complex $X$ on which an RPT complex $A$ already acts, and one is interested in studying multiplications on $X$ which extend to given action of $A$. The absolute theory of RPT-constructions given in [3] does not take care of this problem. One needs a relative theory. The object of this section is to develop such a relative theory.

Suppose $A$ is an RPT-complex of arbitrary rank as in $\$ 1$. Assume that $X$ is two-sided $A$-complex, and let

$$
M_{0}(X) \subset M_{1}(X) \subset \cdots \subset M_{k}(X)
$$

be an ascending sequence of two-sided $A$-complexes such that $M_{0}(X)=A$, $M_{1}(X)=X$, and $M_{p}(X)$ is a subcomplex of $M_{p+1}(X)$ for $k>p \geqq 0$. Suppose also that there is a two-sided invariant map,

$$
\mu_{p, q}: M_{p}(X) \times_{A} M_{q}(X) \rightarrow M_{p+q}(X),
$$

for all integers $p, q \geqq 0$ with $p+q \leqq k$, such that the following conditions are satisfied. 
(3.1) $)_{\mathrm{a}}$ The map $\mu_{p, q} \mid M_{p}(X) \cup M_{q}(X)$ is the folding map;

(3.1) any two maps $\mu_{p, q}$ and $\mu_{p^{\prime}, q^{\prime}}$ agree on their common domain of definition; and

the maps $\mu_{p, q}$ are associative, i.e.

$$
\begin{aligned}
& \mu_{p+q, r}\left(\mu_{p, q} \times 1\right)=\mu_{p, q+r}\left(1 \times \mu_{q, r}\right) \\
& \text { whenever the mappings are defined. }
\end{aligned}
$$

Notice that $M_{k}(X)$ is a two-sided $A$-complex filtered by two-sided $A$-subcomplexes and with a partially defined multiplication which is associative and which extends the action of $A$ on both sides, as (3.1) a easily implies. If $A=\left\{a^{0}\right\}$, then this is precisely the situation described in [3]. Again as in [3], we need another condition on $\mu_{p, q}$ to guarantee that the product of a cell of $M_{p}(X)$ by a cell of $M_{q}(X)$ is also a cell of $M_{p+q}(X)$. So, proceeding in a similar fashion, we note that the mappings $\mu_{p, q-1}$ and $\mu_{p-1, q}$ induce together a cellular mapping

$$
\delta_{p, q}: M_{p} \times_{A} M_{q-1} \cup M_{p-1} \times_{A} M_{q} \rightarrow M_{p+q-1},
$$

where $M_{k}$ stands for $M_{i}(X)$. By definition,

$$
\left[M_{p}(X), M_{q}(X)\right]_{A}=M_{p+q-1}(X) \cup \delta_{p, q} M_{p}(X) \times_{A} M_{q}(X)
$$

where the complex $M_{p}(X) \times_{A} M_{q}(X)$ is attached to $M_{p+q-1}(X)$ by the mapping $\delta_{p, q}$. It is easy to see that $\left[M_{p}(X), M_{q}(X)\right]_{A}$ is a two-sided $A$-complex and that it contains $M_{p+q-1}(X)$ as a two-sided $A$-subcomplex. The desired condition is:

the map $\mu_{p, q}$ induces an imbedding of $\left[M_{p}(X), M_{q}(X)\right]_{A}$ into $M_{p+q}(X)$ as a two-sided $A$-subcomplex, for all $p, q \geqq 0$ and $p-q \leqq k$.

Definition (3.2). Suppose $X$ is a two-sided A-complex, and let

$$
A=M_{0}(X) \subset M_{1}(X)=X \subset \cdots \subset M_{k}(X)
$$

be an ascending sequence of two-sided A-complexes. Assume that for every pair of integers $p, q \geqq 0$ such that $p+q \leqq k$, we are given a two-sided A-mapping,

$$
\mu_{p, q}: M_{p}(X) \times_{A} M_{q}(X) \rightarrow M_{p+q}(X),
$$

subject to the conditions $(3.1)_{\mathrm{a}}, \cdots,(3.1)_{\mathrm{d}}$ given above. Then we say that

$$
\mathscr{M}_{k}(X)=\left(M_{0}(X), \cdots, M_{k}(X) ; \mu_{p, q}, A\right)
$$

is a set of A-relations in $k$ variables.

Note that if $A=\left\{a^{0}\right\}$, then this notion reduces to that of [3]. Thus any set of relations in the old sense is a set of $\left\{a^{0}\right\}$-relations in the sense of Definition (3.2). One can relativize an absolute set of relations in other ways, and sometimes it is both natural and necessary to do so. In fact, let

$$
\mathscr{M}_{k}(X)=\left(M_{0}(X), \cdots, M_{k}(X), \mu_{p, q}\right)
$$


be a set of relations in the sense of [3]. Let $A=X_{\infty}$, the reduced product complex of James [4]. By definition, let

$$
\tilde{M}_{p}=A \cup_{\gamma} A \times M_{p}^{\prime} \times A,
$$

where $M_{p}^{\prime}$ is the complex $A \cup M_{p}(X)$ obtained by attaching $A=X_{\infty}$ to $M_{p}(X)$ along the subcomplex $X_{p}$ and the attaching map

$$
\gamma: A \times A \times A \rightarrow M_{p}^{\prime}
$$

is the one induced by the multiplication in $A$. Observe that $\tilde{M}_{0}=A$. Put $\tilde{X}=\tilde{M}_{1}$ and $M_{p}(\tilde{X})=\tilde{M}_{p}$. Denote by $\tilde{\mu}_{p, q}$ the two-sided invariant maps

$$
\tilde{\mu}_{p, q}: M_{p}(\tilde{X}) \times_{A} M_{q}(\tilde{X}) \rightarrow M_{p+q}(\tilde{X})
$$

induced by $\mu_{p, q}$. Then the set

$$
\mathscr{M}_{k}\left(\tilde{M}_{1}\right)=\left(M_{0}(\tilde{X}), \cdots, M_{k}(\tilde{X}) ; \tilde{\mu}_{p, q}, A\right)
$$

is a set of $A$-relations in $k$ variables equivalent to the original set $\mathscr{M}_{k}(X)$ in the sense that it does not restrict the multiplications $\mu_{p, q}$ any further (see end of section). Clearly one can, in a similar manner, relativize the set $\mathscr{M}_{k}(X)$ with respect to any of the RPT-complexes ${ }^{m} M$ generated by $X$ modulo the subsets

$$
\mathscr{M}_{m}(X)=\left(M_{0}(X), \cdots, M_{m}(X) ; \mu_{p, q}\right), \quad m \leqq k-1 .
$$

Under certain circumstances the converse is also true. We shall indicate how it can be done by discussing a simple situation. Suppose $A=X_{\infty}$, the reduced product complex of a special complex $X$, and assume that

$$
\mathscr{M}_{2}(\tilde{X})=\left(M_{0}(\tilde{X}), M_{1}(\tilde{X}), M_{2}(\tilde{X}) ; \tilde{\mu}_{p, q}, A\right)
$$

is a set of relations on $\tilde{X}$ in two variables such that

$$
\tilde{X}=M_{1}(\tilde{X})=A \cup_{f} A \times D^{r} \times A,
$$

where $D^{r}$ is the $r$-disc and the attaching map

$$
f: A \times S^{r-1} \times A \rightarrow A
$$

extends the multiplication in $A$. Assume also that

$$
M_{2}(\tilde{X})=\left[M_{1}(\tilde{X}), M_{1}(\tilde{X})\right]_{A} .
$$

To define an equivalent absolute set of relations, let $k$ be the smallest integer such that $f\left(S^{r-1}\right) \subset X_{k}$, and put

$$
M_{k}(X)=X_{k} \cup_{f} D^{r}
$$

Then the set

$$
\mathscr{M}_{k}(X)=\left(M_{0}(X), \cdots, M_{k}(X) ; \mu_{p, q}\right),
$$


where $M_{0}=x^{0}$, the 0 -cell of $X, M_{1}=X, M_{p}=X_{p}$, for $p \leqq k-1, M_{k}$ is as above, and $\mu_{p, q}$ are the natural maps, is a set of relations in the absolute sense (see [3]) equivalent to the given set $\mathscr{M}_{2}(\tilde{X})$.

Definition (3.3). Suppose that

$$
\begin{aligned}
& \mathscr{M}_{k}(X)=\left(M_{0}(X), \cdots, N_{k}(X) ; \mu_{p, q}, A\right) \text { and } \\
& \mathscr{M}_{k}\left(X^{\prime}\right)=\left(M_{0}\left(X^{\prime}\right), \cdots, M_{k}\left(X^{\prime}\right) ; \mu_{p, q}^{\prime}, A^{\prime}\right)
\end{aligned}
$$

are two sets of two-sided $A$-and $A^{\prime}$-relations on $X$ and $X^{\prime} . A$ mapping

$$
f: \mathscr{M}_{k}(X) \rightarrow \mathscr{M}_{k}\left(X^{\prime}\right)
$$

is a tuple of two-sided invariant maps $\left(f_{0}, \cdots, f_{k}\right)$ such that

$$
\mu_{p, q}^{\prime}\left(f_{p} \times f_{q}\right)=f_{p+q} \mu_{p, q}
$$

for all $p$ and $q$ such that $p+q \leqq k$.

Again this concept reduces to that of [3] in the case $A=\left\{a^{0}\right\}$.

Suppose next that $M$ is an $H$-complex with multiplication $\mu$, and assume that $M$ contains an RPT-complex $A$ of arbitrary rank as a sub $H$-complex. Suppose also that $M$ is filtered by an ascending sequence of two-sided $A$-complexes

$$
A=M_{0} \subset M_{1} \subset \cdots \subset M_{k} \subset \cdots,
$$

and assume that $\mu$ takes $M_{p} \times_{A} M_{q}$ into $M_{p+q}$, for all $p$ and $q \geqq 0$. Denote $M_{1}$ by $X$, and write $M_{p}(X)$ for $M_{p}$ for all $p \geqq 0$. Then, as in the absolute case (see $\S 1$ ), we say that $M$ is an $R P T$-complex relative to $A$ if, and only if, the set

$$
\mathscr{M}_{k}(X)=\left(M_{0}(X), M_{1}(X), \cdots, M_{k}(X) ; \mu_{p, q}, A\right)
$$

where $\mu_{p, q}$ is induced by $\mu$ on $M_{p}(X) \times_{A} M_{q}(X)$ is a set of $A$-relations on $X$, in the above sense, for all integers $k \geqq 2$. If, moreover, there is an integer $m$ such that

$$
\mathscr{M}_{p}(X)=\bigcup \mu_{r, s}\left(M_{r}(X) \times_{A} M_{s}(X)\right), \quad r, s>0, r+s=p,
$$

for all $p>m$, then we say that $M$ is of rank $\leqq m$. Otherwise we say that $M$ is of infinite rank. Here also an RPT-complex relative to $\left\{a^{0}\right\}$ is just an RPT-complex in the absolute sense, as defined in the first section.

Next we wish to show that every set of $A$-relations generates an RPT-complex relative to $A$. The construction will be seen to be a modification of the one given in [3] for the absolute case.

Suppose that

$$
\mathscr{M}_{k}(X)=\left(M_{0}(X), \cdots, M_{k}(X) ; \mu_{p, q}, A\right)
$$

is a set of relations as above, where $\boldsymbol{A}$ is an arbitrary RPT-complex. By definition, the index of an element $a$ in $A$ is 0 , and the index of an element $x$ in $M_{i}-M_{i-1}$ is $i$. If 


$$
u=\left(x_{1}, \cdots, x_{n}\right) \in\left(M_{k}\right)^{n},
$$

where $\left(M_{k}\right)^{n}$ is the $n$-fold cartesian product $M_{k} \times M_{k} \times \cdots \times M_{k}$, then the index of $u$ is $\sum_{i=1}^{n}$ index $\left(x_{i}\right)$. Consider now the set

$$
S_{n}=\left\{u \in\left(M_{k}\right)^{n} \mid \operatorname{index}(u) \leqq n\right\} .
$$

It can be easily seen that $S_{n}$ is a two-sided $A$-subcomplex of $\left(M_{k}\right)^{n}$. If $u=\left(x_{1}, \cdots, x_{n}\right)$ and $u^{\prime}=\left(x_{1}^{\prime}, \cdots, x_{n}^{\prime}\right)$ are in $\left(M_{k}\right)^{n}$, then $u^{\prime}$ is said to be an $\operatorname{amalgam}\left({ }^{2}\right)$ of $u$ if, and only if, there is an integer $p<n$ such that

$$
\begin{aligned}
x_{q} & =x_{q}^{\prime}, \text { if } q<p, \text { or } q>p+1, \\
\mu\left(x_{p}, x_{p+1}\right) & =x_{p}^{\prime}, \text { and } \\
a^{0} & =x_{p+1}^{\prime},
\end{aligned}
$$

where $a^{0}$ is the 0 -cell of $A$ and $\mu$ stands for the appropriate multiplication of $\mathscr{M}_{k}(X)$. The notion of amalgamation generates an equivalence relation on $S_{n}$ (see [3]). Denote the quotient space by $M_{n}^{\prime}(X)$, and give it the quotient topology. Note that $A$ acts on the left and right on $M_{n}^{\prime}(X)$ and that the two actions commute with each other. Since the mappings $\mu_{p, q}$ are associative, the juxtaposition map of $S_{m} \times S_{n}$ into $S_{m+n}$ induces a continuous mapping

$$
\mu_{r, s}^{\prime}: M_{r}^{\prime}(X) \times_{A} M_{s}^{\prime}(X) \rightarrow M_{r+s}^{\prime}(X) .
$$

Note that the mappings $\mu_{p, q}^{\prime}$ induce a mapping

$$
\delta_{r, s}: M_{r}^{\prime}(X) \times_{A} M_{s-1}^{\prime}(X) \cup M_{r-1}^{\prime}(X) \times_{A} M_{s}^{\prime}(X) \rightarrow M_{r+s-1}^{\prime}(X)
$$

By definition, let

$$
\left[M_{r}^{\prime}(X), M_{s}^{\prime}(X)\right]_{A}=M_{r+s-1}^{\prime}(X) \cup_{\delta_{r}, s} M_{r}^{\prime}(X) \times_{A} M_{s}^{\prime}(X) .
$$

Then $A$ acts on both sides of $\left[M_{r}^{\prime}(X), M_{s}^{\prime}(X)\right]_{A}$, and the actions commute with each other.

THEOREM (3.6) $r, s^{\cdot} \quad M_{n}^{\prime}(X)$ inherits naturally from $S_{n}$ a cellular structure which makes it a two-sided $A$ complex with the following properties:

(a) $)_{n}$ there is a natural imbedding $\beta_{n-1}$ of $M_{n-1}^{\prime}(X)$ in $M_{n}^{\prime}(X)$ as a two-sided A-subcomplex;

(b) $)_{n}$ if $r+s=n$, then the map $\mu_{r, s}^{\prime}$ of $(3.4)_{r, s}$ is cellular, and $\left[M_{r}^{\prime}(X), M_{s}^{\prime}(X)\right]_{A}$ is a two-sided A-complex imbedded in $M_{n}^{\prime}(X)$ as a two-sided A-subcomplex, the imbedding being induced by $\mu_{r, s}^{\prime}$ of (3.4)

(c) $M_{n}^{\prime}(X)$ is naturally isomorphic to $M_{n}(X)$ of $\mathscr{M}_{k}(X)$ as a two-sided A-complex for $n \leqq k$, and $\mu_{r, s}^{\prime}$ is equal to $\mu_{r, s}$ of $\mathscr{M}_{k}(X)$ as a two-sided invariant map for $r+s \leqq k$.

(2) Cf. definition of $A$-cartesian product in $\$ 1$. 
Note that this theorem is just Theorem (2.4)n of [3], when $A=\left\{a^{0}\right\}$. The proof is also similar to that case, and therefore will not be given.

From here on write $M_{p}(X)$ and $\mu_{r, s}$ for $M_{r}^{\prime}(X)$ and $\mu_{r, s}^{\prime}$, and identify $\left[M_{r}(X), M_{s}(X)\right]_{A}$ with its image in $M_{r+s}(X)$.

Corollary (3.7). Suppose $n>k \geqq r \geqq 1$. Then, if $r<s$,

$$
\left[M_{n-s}, M_{s}\right]_{A} \cap\left[M_{n-r}, M_{r}\right]_{A}
$$

is isomorphic to $\left[M_{n-s}, M_{s-r}, M_{r}\right]_{A}$. Moreover, $M_{n}$ is the union of the twosided A-complexes $\left[M_{n-r}, M_{r}\right]_{A}$, for $r=1, \cdots, k$.

By definition, let

$$
M(X)=\lim _{n} M_{n}(X)
$$

Then $M(X)$ is certainly a two-sided $A$-complex, and the two-sided invariant mappings $\mu_{r, s}^{\prime}$ of (3.4) $)_{r, s}$ induce a two-sided invariant map

$$
\mu: M(X) \times_{A} M(X) \rightarrow M(X) .
$$

THEOREM (3.8). $M(X)$ with the multiplication $\mu$ is an RPT-complex relative to $A$.

Suppose next that $B$ is an associative $H$-space on which $A$ acts on both sides; i.e., the left and right actions of $A$ commute with each other and are associative, and the 0-cell of $A$ acts like the identity. (If $B$ is an $H$-complex, then the product of a cell in $B$ by a cell in $A$ is not required to be a cell of $B$.) Then by a map

$$
f: \mathscr{M}_{k}(X) \rightarrow B
$$

of $\mathscr{M}_{k}$ into $B$, we mean a tuple of two-sided invariant maps $\left(f_{0}, \cdots, f_{k}\right)$, where $f_{p}$ takes $M_{p}(X)$ to $B$, and

$$
f_{p}=\beta\left(f_{q} \times f_{r}\right), \quad p=q+r,
$$

$\beta$ being the multiplication in $B$.

THEOREM (3.9). Suppose that

$$
\mathscr{M}_{k}(X)=\left(M_{0}(X), \cdots, M_{k}(X), \mu_{p, q}, A\right)
$$

is a set of two-sided A-relations on $X$, and suppose that

$$
f: \mathscr{M}_{k}(X) \rightarrow B
$$

is a map of the set $\mathscr{M}_{k}(X)$ into the associative $H$-space $B$ on which $A$ acts on both sides. Then there is a two-sided invariant homomorphism,

$$
f_{\infty}: M(X) \rightarrow B,
$$


uniquely determined by $f$, where $M(X)$ is the RPT-complex generated by $X$ modulo $\mathscr{M}_{k}(X)$. Moreover, $f_{\infty}$ is cellular if $f$ is cellular and $B$ is an H-complex.

The proof is straightforward.

In the first part of the section we indicated how the absolute and relative relations correspond to each other. Suppose

$$
\mathscr{M}_{2}(X)=\left(M_{0}(X), M_{1}\right) X,\left(M_{2}(X) ; \mu_{p, q}\right)
$$

is a set of absolute relations; i.e., $M_{0}(X)=\left\{a^{0}\right\}$ and $M_{1}(X)=X$. Assume also that $M_{2}(X)=X_{2} \cup D^{n}$, where $X_{2}$ is the 2-fold reduced product of $X$ and $D^{n}$ is the $n$-disc. Then, as above, $\mathscr{M}_{2}(X)$ corresponds to a set of $A$-relations

$$
\mathscr{M}_{2}(\tilde{X})=\left(M_{0}(\tilde{X}), M_{1}(\tilde{X}), M_{2}(\tilde{X}) ; \tilde{\mu}_{p, q}, A\right)
$$

where $A=X_{\infty}, \quad M_{1}(\tilde{X})=A \cup A \times D^{n} \times A$, and $M_{2}(\tilde{X})=\left[M_{1}(\tilde{X}), M_{1}(\tilde{X})\right]_{A}$. Here $M_{1}(\tilde{X})$ is formed by attaching $A \times D^{n} \times A$ to $A$ by the multiplication $A \times A \times A \rightarrow A$. One can see easily that the RPT-complex $M(X)$ generated by $X$ modulo $\mathscr{M}_{2}(X)$ is naturally isomorphic to the RPT-complex $M(\tilde{X})$ generated by $\tilde{X}$ modulo $\mathscr{M}_{2}(\tilde{X})$.

4. The universal quasi-fibration of an RPT-complex. One knows that an associative $H$-space $A$ always has a principal quasi-fibration

$$
p_{A}: E(A) \rightarrow B(A)
$$

such that the total space $E(A)$ is aspherical [1]. (Recall that such a principal quasi-fibration is called universal.) This quasi-fibration is, however, not quite appropriate to the needs of this study, since it is not possible in general to tell if the restriction of a quasi-fibration to a subset of the base is also a quasi-fibration. The aim of this section is to construct a universal quasi-fibration (for any RPTcomplex) which is more appropriate in that respect for the cases under consideration here (see Corollary (4.5) below). The construction is based on the following theorem.

Suppose $A$ is an RPT-complex of any rank (see $§ 1$ ), and assume that

$$
\bar{f}: \bar{L} \rightarrow A
$$

is a cellular map of some special complex $L$ into $A$. By definition, let

$$
M_{0}(X)=A \text {, and } M_{1}(X)=X=A \cup A \times A^{\prime} \times A,
$$

where $A^{\prime}=A \cup_{\bar{f}} T L(T L$ being the cone on $L)$ and $A \times A^{\prime} \times A$ is attached to $A$ by the multiplication $A \times A \times A \rightarrow A$. Clearly $M_{0}(X)$ and $M_{1}(X)$ are two-sided $A$-complexes; and, if we put $M_{2}(X)=\left[M_{1}(X), M_{1}(X)\right]_{A}$, then the set

$$
\mathscr{M}_{2}(X)=\left(M_{0}(X), M_{1}(X), M_{2}(X) ; \mu_{p, q}, A\right),
$$


where $\mu_{p, q}$ are the natural maps, is a set of $A$-relations in the sense of $\S 3$. Suppose $M(X)$ is the RPT-complex generated by $X$ modulo $\mathscr{M}_{2}(X)$. Assume now that $E(A)$ is a contractible right $A$-complex, and denote $E(A) / / A$ by $B(A)$. Then

$$
p_{A}: E(A) \rightarrow B(A)
$$

( $p_{A}$ being the natural projection) is a principal quasi-fibration according to Theorem (2.2). We wish to imbed $E(A)$ in a contractible left $M(X)$-complex $E(M)$ in such a way that the action of $M$ on $E(M)$ extends the action of $A$ on $E(A)$. Consider, therefore, the right $M$-complex $E(A) \times{ }_{A} M$, and let

$$
p_{A}^{\prime}: E(A) \times_{A} M \rightarrow B(A)
$$

be the quasi-fibration induced by the projection on the first factor. Observe that we can extend $f$ to a map

$$
g: L=S \# L \rightarrow E(A) \times{ }_{A} M
$$

which is equal to the natural map $g_{+}$of $T L$ into $M \subset E(A) \times{ }_{A} M$ on the upper cone and to a contraction of $\bar{f}$,

$$
g_{-}:(T L, L) \rightarrow(E(A), A),
$$

on the lower cone. ( $S \# L$ is the suspension of $L$.) Then

$$
E(M)=E(A) \times_{A} M \cup_{\beta} T L \times M,
$$

where the attaching map,

$$
\beta: L \times M \rightarrow E(A) \times_{A} M,
$$

is the map that takes $(x, y)$ to $g(x) y$ and is clearly a right $M$-complex, and the natural projection,

$$
p_{M}: E(M) \rightarrow B(M)=E(M) / / M
$$

is a principal quasi-fibration for $M$. Observe also that

$$
B(M)=B(A) \cup_{\gamma} T L,
$$

where $\gamma$ is the composition of $g$ and $p_{A}^{\prime}$.

TheOREM (4.4). Suppose $E(A)$ is a contractible right A-complex where $A$ is a given RPT-complex, and let $M$ be the RPT-complex relative to $A$ which is determined by (4.1). Then the complex $E(M)$ of (4.2) is a contractible right $M$-complex which contains $E(A)$ as a subcomplex, the imbedding being of a right A-complex into a right M-complex. Moreover, in the induced diagram 


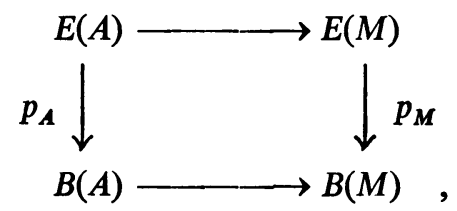

the vertical maps are universal quasi-fibrations, and $B(M)$ is obtained from $B(A)$ according to (4.3).

We need prove only that $E(M)$ is contractible, which we shall do a bit later.

Corollary (4.5). Suppose $A$ is an RPT-complex. Then there is a universal quasi-fibration,

$$
p_{A}: E(A) \rightarrow B(A) \text {. }
$$

Moreover, if $N$ is an RPT-subcomplex of $A$, then $E(A), B(A)$ and $p_{A}$ can be constructed so that

$$
p_{A}^{-1}(K)=E(N) \times{ }_{N} A,
$$

where $K$ is a subcomplex of $B(A)$ and

$$
\left(p_{A} \mid E(N)\right): E(N) \rightarrow K
$$

is a universal quasi-fibration for $N$.

The corollary can be proved easily by expressing $A$ as the ascending sequence of RPT-complexes

$$
{ }^{2} A \subset{ }^{3} A \subset \cdots \subset{ }^{k} A \subset \cdots,
$$

where ${ }^{2} A=X_{\infty}$, for some special complex ( $X_{\infty}$ being the reduced product of James), and ${ }^{k} A$ is obtained from ${ }^{k-1} A$ by adding one indecomposable cell to ${ }^{k-1} A$, in the same manner that the $R P T$-complex $M(X)$ is obtained from $A$ by adding the cone on $\bar{L}$ (see (4.1)). Now, by definition, let

$$
E\left({ }^{2} A\right)={ }^{2} A \cup T X \times{ }^{2} A,
$$

where $T X$ is the cone on $X$ and $T X \times{ }^{2} A$ is attached by ${ }^{2} \alpha \mid X \times{ }^{2} A\left({ }^{2} \alpha\right.$ being the multiplication in $\left.{ }^{2} A\right)$. It is easy to see that $E\left({ }^{2} A\right)$ is contractible; and, if we make ${ }^{2} A$ act on the right on $E\left({ }^{2} A\right)$, then $E\left({ }^{2} A\right)$ becomes a right ${ }^{2} A$-complex, and

$$
p_{2}: E\left({ }^{2} A\right) \rightarrow E\left({ }^{2} A\right) /{ }^{2} A=B\left({ }^{2} A\right)=S \# X
$$

is a universal quasi-fibration for ${ }^{2} A$. Now, by Theorem (4.4), we can find an ascending sequence 


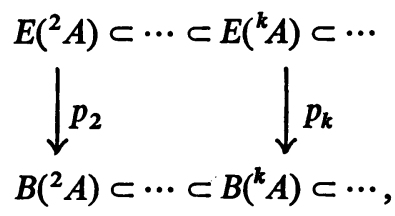

where $p_{k}$ is a universal quasi-fibration for ${ }^{k} A$.

By definition, let

$$
E(A)=\lim _{k} E\left({ }^{k} A\right), B(A)=\lim _{k} B\left({ }^{k} A\right), \text { and } p_{A}=\lim _{k} p_{k} .
$$

Then the map

$$
p_{A}: E(A) \rightarrow B(A)
$$

is a universal quasi-fibration for $A$ (see (2.1) of [2]).

The fact that $E(A)$ can be constructed so as to satisfy the second assertion of the corollary can be shown by constructing $E(N)$ first and then applying Theorem (4.4) to a filtration of $\boldsymbol{A}$ by RPT-subcomplexes starting with $N$ and such that each RPT-subcomplex is obtained from the preceding one by adding one indecomposable cell only.

Similarly one can construct a universal quasi-fibration on which $A$ acts on the left.

Proof of Theorem (4.4). As we mentioned above, we need prove only that $E(M)$ is contractible. So let $P(B(M))$ be the space of Moore paths based at the 0 -cell $b^{0}$, and let

$$
q: P(B(M)) \rightarrow B(M)
$$

be the fibration that sends a path to its end-point. The fiber is the space of loops $\Omega(B(M))$. Observe that $\Omega(B(M))$ is an associative $H$-space and that it acts on the right on $P(B(M)$ ). We can find a homomorphism,

$$
u: M \rightarrow \Omega(B(M)),
$$

which is a homotopy equivalence [3]. It is not hard to see that $u$ can be extended to a map

$$
v: E(M) \rightarrow P(B(M))
$$

of an $M$-space into an $\Omega(B(M))$-space such that the induced diagram

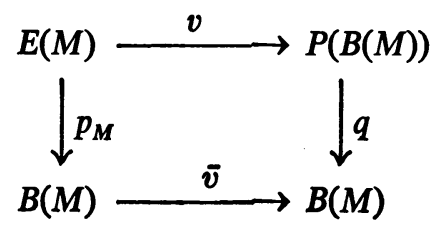


is commutative and the induced map $\bar{v}$ is homotopic to the identity. This implies that $E(M)$ is contractible, which is what is to be proved.

REMARK (4.6). Given a simply-connected complex $B$, one can find an RPTcomplex $A$ and a homomorphism $\phi$ of $A$ into $\Omega(B)$ which is a homotopy equivalence. It is easy to see that

$$
B \sim B(A) .
$$

Thus the universal quasi-fibration represents the canonical fibration of the space of paths over $B$.

Next we show that the principal quasi-fibrations

$$
p: N \rightarrow N / / A
$$

admits a classifying map, where $N$ is a right $A$-complex. Let

$$
p_{A}: E(A) \rightarrow B(A)
$$

be a universal quasi-fibration on which $A$ acts on the left, constructed as above. Consider the commutative diagram,

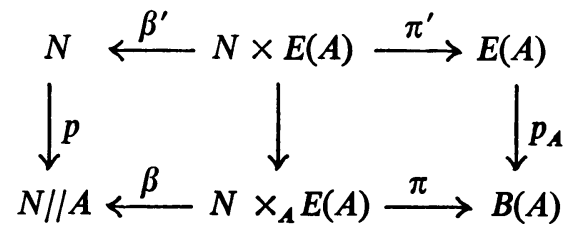

where the vertical maps are the natural projections and the horizontal maps are the projections on the factors and the maps induced by them.

Theorem (2.2)' implies that

$$
\begin{aligned}
& \beta: N \times_{A} E(A) \rightarrow N / / A \text { and } \\
& \pi: N \times_{A} E(A) \rightarrow B(A)
\end{aligned}
$$

are quasi-fibrations with fibers $E(A)$ and $N$ respectively. Since $E(A)$ is contractible, $\beta$ has a homotopy-inverse, $\beta^{-1}$, say. Define

$$
\chi=\beta^{-1} \pi: N / / A \rightarrow B(A)
$$

to be the classifying map of the quasi-fibration

$$
p: N \rightarrow N / / A \text {. }
$$

5. Fibrations of complexes by subcomplexes. Let us say that a sequence

$$
\mathscr{F}: F \stackrel{i}{\longrightarrow} B \stackrel{f}{\longrightarrow} Y
$$

of special complexes and cellular maps is a fiber sequence, if and only if, $\mathscr{F}$ can be imbedded in a homotopy-commutative diagram, 


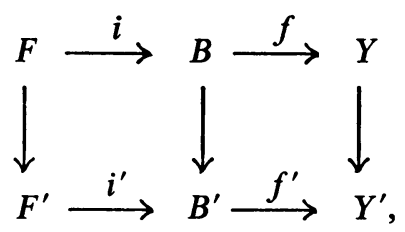

where the lower row is a Serre fibration and the vertical maps are all homotopyequivalences. $F$ is the fiber, $B$ the total space, and $Y$ the base-space of $\mathscr{F}$.

Consider now the following problem, which is essentially Problem 10 in Massey's list of 1955 [6]: suppose

$$
i: F \rightarrow B
$$

is a map of special complexes. When is it possible to imbed in a fiber sequence

$$
\mathscr{F}: F \stackrel{i}{\longrightarrow} B \stackrel{f}{\longrightarrow} Y \text { ? }
$$

\section{Is $\mathscr{F}$ unique?}

Assume $i$ is an imbedding, since there is no loss of generality in doing so. One can see right away that the problem does not always have a solution. For example, let $F=S^{2 n}$, the $2 n$-sphere with $n>0, B=S^{2 n+1}$, the $(2 n+1)$-sphere, and let $i: S^{2 n} \rightarrow S^{2 n+1}$ be the natural imbedding. Since $S^{2 n}$ cannot be an $H$-space, $i$ cannot be imbedded in a fiber sequence [10]. Note also that the injection of $F$ in $B$ plays an important role. In fact, for some spaces $F$ and $B$ it may happen that one imbedding gives rise to a fiber sequence, while another does not. For example, let $B=T F \times F$, where $T F$ is the cone on $F$. Then if we imbed $F$ in $B$ as the second factor, the projection on the first factor defines a fiber sequence for which $F$ is the fiber; but if we imbed $F$ as the base of the second factor $T F$, then the resulting sequence is not a fiber sequence, for this would imply that $F$ was an $H$-space [10].

Moreover, the sequence

$$
\mathscr{F}: F \stackrel{i}{\longrightarrow} B \stackrel{f}{\longrightarrow} Y
$$

in which $i$ can be imbedded is not unique. For example, let $F=S^{3}$, the 3-sphere, and $B=S^{7}$, the 7-sphere; and let $i$ be the natural imbedding. Then there are inequivalent multiplications $\{\alpha\}$, on $S^{3}$, which yield inequivalent fiber sequences

$$
\alpha: S^{3} \rightarrow S^{7} \rightarrow S^{4},
$$

according to [5].

We shall give necessary and sufficient conditions for the problem to have a solution, as well as a method, at least in theory, for obtaining all solutions when $B$ and $F$ are simply connected special complexes and $F$ is a subcomplex of $B$. Denote the space of Moore paths based at $b^{0}$ by $P(B)$ and by $\Omega(B)$ the subspace 
of loops. Then, with the usual juxtaposition multiplication, $P(B)$ is a right $\Omega(B)$ space. Suppose

$$
u: A \rightarrow \Omega(B)
$$

is a homomorphism of some RPT-complex $A$ into $\Omega(B)$, which is a homotopy equivalence [3]. Then, as we saw in $\S 4$, there is a commutative diagram,

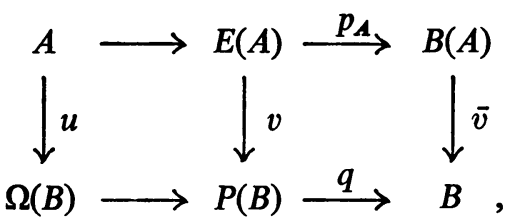

where the upper row is the universal quasi-fibration for $A$ and the lower row is the canonical fibration which sends a path to its end-point and $v$ is a map of a right $A$-space into a right $\Omega(B)$-space. Moreover, $u, v$, and $\bar{v}$ are all homotopy equivalences. Now, let

$$
U=p_{A}^{-1} \bar{v}^{-1}(F) \text {, and } \Omega(B, F)=q^{-1}(F) .
$$

Corollary (4.5) of $\$ 4$ implies that the restriction of $p_{A}$ to $U$ is a quasi-fibration, and hence, comparing the homotopy groups, we conclude that

$$
\left(v \mid p_{A}^{-1} \bar{v}^{-1}(F)\right): U \rightarrow \Omega(B, F)
$$

is a homotopy equivalence. (Recall that $\Omega(B, F)$ is of the homotopy type of a countable CW-complex [8].)

What kind of $A$-relations does $U$ admit, when $F$ is the fiber for some fiber sequence in which the total space is $B$ ? The following theorem gives the answer.

THEOREM (5.3). Suppose that

$$
F \stackrel{i}{\longrightarrow} B \stackrel{f}{\longrightarrow} Y
$$

is a fiber sequence such that $B$ and $F$ are simply connected special complexes and $F$ is a subcomplex imbedded by $i$ in $B$. Assume also that $A$ is the RPT-complex representing $\Omega(B)$ and $U$ the right $A$-complex representing $\Omega(B, F)$ as in (5.1) and (5.2). Then there is a right invariant map,

$$
\phi: U \rightarrow M_{1},
$$

which is a homotopy equivalence, and, for every $n \geqq 1$, there is a set of A-relations on $M_{1}$,

$$
\mathscr{M}_{n}\left(M_{1}\right)=\left(M_{0}, \cdots, M_{n} ; \mu_{p, q}, A\right),
$$

such that 
(a) $\mathscr{M}_{k}\left(M_{1}\right)$ is a subset of $\mathscr{M}_{n}\left(M_{1}\right)$, for $k<n$, and

(b) ${ }_{n}$ there is a two-sided invariant deformation retraction,

$$
{ }^{k} h_{t}: M_{k} \rightarrow M_{k}, k=2, \cdots, n,
$$

of $M_{k}$ onto $M_{k-1}$.

The proof will be given in $\$ 7$ below.

Definition (5.4). Suppose that $M_{1}$ is a two-sided A-complex, $A$ being an $R P T$-complex. Then $M_{1}$ is said to admit a $Q_{n}$-structure if and only if, there is a set of A-relations on $M_{1}$,

$$
\mathscr{M}_{n}\left(M_{1}\right)=\left(M_{0}, \cdots, M_{n} ; \mu_{p, q}, A\right),
$$

subject to condition (b) ${ }_{n}$ of Theorem (5.3).

Note that the necessary conditions of Theorem (5.3), which are satisfied if $F$ is the fiber of a fiber sequence for which $B$ is the total space, are of two kinds. First, $U$ or, equivalently, $\Omega(B, F)$ must be of the homotopy type of a two-sided $A$-complex $M_{1}$; and second, $M_{1}$ must admit a $Q_{n}$-structure for all $n \geqq 2$, and the $Q_{n}$-structures must extend each other. We shall take up the discussion of when $M_{1}$ exists in another paper, confining our attention here to the implications of a $Q_{n}$-structure. We shall see in effect that the existence of a $Q_{n}$-structure is a sufficient condition for the existence of sequences,

$$
\mathscr{F}_{n}: F \stackrel{i}{\longrightarrow} B \stackrel{f}{\longrightarrow} B_{n},
$$

which are fiber sequences in a range which widens with $n$. The following condition will be needed in most of what follows.

Condition (5.5). Suppose $B$ and $F$ are simply connected special complexes with $F$ a subcomplex of $B$, and let $A$ and $U$ be the RPT-complex and the right $A$-complex representing $\Omega(B)$ and $\Omega(B, F)$, as in (5.1) and (5.2). The condition is that there be a two-sided A-complex $M_{1}$ and a right $A$-map

$$
\phi: U \rightarrow M_{1}
$$

which is a homotopy equivalence.

Consider now the set of $A$-relations

$$
\mathscr{M}_{n}\left(M_{1}\right)=\left(M_{0}, \cdots, M_{n} ; \mu_{p, q}, A\right),
$$

and denote by ${ }^{k} M$ the RPT-complex relative to $A$ which is generated by $M_{1}$ modulo the subset $\mathscr{M}_{k}\left(M_{1}\right)$ of $\mathscr{M}_{n}\left(M_{1}\right)$. Put $B_{k}=B\left({ }^{k} M\right)$, the base-space of the universal quasi-fibration of ${ }^{k} M$ (see $\S 4$ ). Then the imbedding of ${ }^{k-1} M$ into ${ }^{k} M$ induces an imbedding

$$
\eta_{k}: B_{k-1} \rightarrow B_{k}
$$


Thus we obtain an ascending sequence

$$
B \rightarrow B_{1} \rightarrow \cdots \rightarrow B_{n}
$$

of special complexes. Let

and

$$
f_{k}=\eta_{k} \cdots \eta_{1}: B \rightarrow B_{k}
$$

$$
F_{k}=\Omega\left(B_{k}, B\right)=\left\{\alpha \in \tilde{P}\left(B_{k}\right) \mid \alpha(0) \in B\right\},
$$

where $\widetilde{P}\left(B_{k}\right)$ is the space of Moore paths $\alpha$ ending in $b^{0}$ (i.e., $\alpha(1)=b^{0}$ ). Suppose that

$$
i_{k}: F_{k} \rightarrow B
$$

is the natural map which sends a path to its initial point. Then

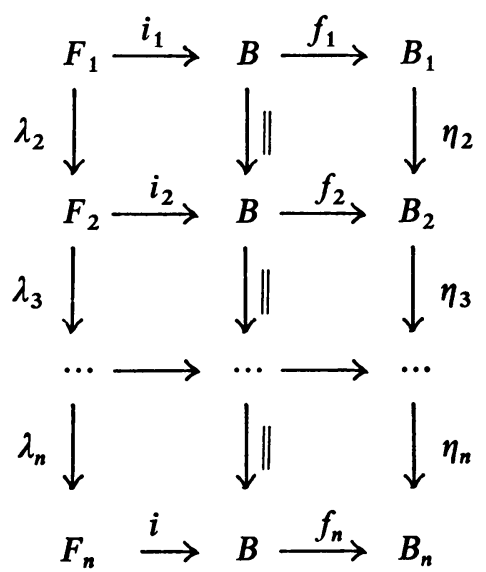

is a commutative diagram in which the rows are fiber sequences, and $\lambda_{j}$ is the natural imbedding of $F_{j-1}$ into $F_{j}$.

THEOREM (5.7). Suppose that Condition (5.5) is satisfied. Assume that the set of A-relations (5.6) defines a $Q_{n}$-structure on $M_{1}$, the two-sided A-complex of (5.5). Then, for $k \leqq n-1$, with the notation as in the preceding paragraph, there are natural maps,

$$
\gamma_{k}: F \rightarrow F_{k} \text { and } \beta_{k}: F_{k} \rightarrow F,
$$

for $k=1, \cdots, n$, such that

(a) $\beta_{k} \gamma_{k} \sim 1, \lambda_{k} \gamma_{k} \sim \gamma_{k+1}$, and $g i=i_{k} \gamma_{k}$, where $g$ is a homotopy equivalence of $B$; and

(b) $)_{k}$ the induced homomorphism

$$
\left(\gamma_{k}\right)_{*}: \pi_{j}(F) \rightarrow \pi_{j}\left(F_{k}\right)
$$


is an isomorphism for $j \leqq c(k+1)+(k-2)$, where $(c-1)$ is the connectivity of $F$. ( denotes homotopy.)

The proof is given in $\$ 9$.

Thus the existence of a $Q_{n}$-structure on $M_{1}$ implies that the pair $(B, F)$ can be "approximated" by pairs $\left(B, F_{n}\right)$ with the property that $F_{n}$ is a fiber in a fiber sequence in which $B$ is the total space and $F$ and $F_{n}$ agree in a range which increases with $n$. Thus the existence of $Q_{n}$-structures on $\Omega(B, F)$ which extend each other for all $n$ is a sufficient condition for the existence of a fiber sequence

$$
F \rightarrow B \rightarrow X \text {. }
$$

Note that if $B$ is contractible, then the fibers $F_{k}$ are all of the homotopy type of loopspaces [9], and, hence, $F$ is increasingly getting like a loopspace. This suggests that if $B$ is contractible, our conditions are just Stasheff's $A_{n}$-structures [11]. In fact, this is the case. First we need prove that Condition (5.5) is satisfied.

(5.8). Suppose $B$ is a contractible special complex and $F$ is a simply connected subcomplex, and let $A$ and $U$ be the RPT-complex and the right A-complex representing $\Omega(B)$ and $\Omega(B, F)$, as in (5.1) and (5.2).

Then there is a right A-map,

$$
\phi: U \rightarrow M_{1},
$$

of $U$ into a two-sided A-complex $M_{1}$ which is a homotopy equivalence.

In fact, let

$$
M_{1}=U \cup_{\beta} A \times U
$$

where the attaching map is the map

$$
\beta: A \times A \cup U \rightarrow U
$$

induced by the multiplication in $A$. Then clearly $M_{1}$ is a two-sided $A$-complex, and the right $A$-imbedding,

$$
\phi: U \rightarrow M_{1},
$$

is a homotopy equivalence.

Let us denote by $\bar{M}_{1}$ the complex $M_{1}$ without the action of $A$. (Recall that an $A_{n}$-structure in the sense of Stasheff is according to [3], just a $Q_{n}$-structure relative to $\left\{a^{0}\right\}, a^{0}$ being the 0 -cell of $A$.)

THEOREM (5.9). Suppose that the assumptions of (5.8) are satisfied, and let $\bar{M}_{1}$ and $M_{1}$ be as above. Assume that

$$
\mathscr{M}_{n}\left(\bar{M}_{1}\right)=\left(\bar{M}_{0}, \cdots, \bar{M}_{n} ; \bar{\mu}_{p, q},\left\{a^{0}\right\}\right)
$$


is a set of $\left\{a^{0}\right\}$-relations which defines an $A_{n}$-structure on $\bar{M}_{1}$. Then there is a set of A-relations

$$
\mathscr{M}_{n}\left(M_{1}\right)=\left(M_{0}, \cdots, M_{n} ; \mu_{p, q}, A\right)
$$

which defines a $Q_{n}$-structure on $M_{1}$, and conversely. In either case, there is a map

$$
f_{n}: \mathscr{M}_{n}\left(\bar{M}_{1}\right) \rightarrow \mathscr{M}_{n}\left(M_{1}\right)
$$

which is a homotopy equivalence when restricted to $\bar{M}_{1}$.

We shall sketch the proof in the case $n=2$ only, the general case being similar. So suppose that

$$
\mathscr{M}_{2}\left(M_{1}\right)=\left(M_{0}, M_{1}, M_{2} ; \mu_{p, q}, A\right)
$$

is a set of $A$-relations which defines a $Q_{2}$-structure on $M_{1}$ (see Definition (5.4)). By definition, let

$$
\bar{M}_{0}=\left\{a^{0}\right\} \text { and } \bar{M}_{1}=M_{1} \text {, }
$$

and let

$$
\gamma:\left[\bar{M}_{1}, \bar{M}_{1}\right] \rightarrow\left[M_{1}, M_{1}\right]_{A}
$$

be the natural projection. Let

$$
\bar{M}_{2}=M_{2} \cup_{\gamma}\left[\bar{M}_{1}, \bar{M}_{1}\right] \times I,
$$

where $I$ is the unit interval, the point $(x, 1)$ is identified with $\gamma(x) \in M_{2}$, and $\left\{a^{0}\right\} \times I$ is identified with the basepoint $a^{0} \cdot\left(\bar{M}_{2}\right.$ is just the mapping cylinder of $\gamma$ with the segment through $a^{0}$ identified with $a^{0}$.) Clearly the set

$$
\mathscr{M}_{2}\left(\bar{M}_{1}\right)=\left(\bar{M}_{0}, \bar{M}_{1}, \bar{M}_{2} ; \bar{\mu}_{p, q},\left\{a^{0}\right\}\right)
$$

where $\bar{\mu}_{p, q}$ are the natural maps is a set of relations which defines an $A_{2}$-structure on $\bar{M}_{1}$, and the natural map

$$
f_{2}: \mathscr{M}_{1}\left(\bar{M}_{2}\left(\bar{M}_{1}\right) \rightarrow \mathscr{M}_{2}\left(M_{1}\right)\right.
$$

is a map which satisfies the requirements of the theorem.

The converse is similar. Suppose that

$$
\mathscr{M}_{2}\left(\bar{M}_{1}\right)=\left(\bar{M}_{0}, \bar{M}_{1}, \bar{M}_{2} ; \bar{\mu}_{p, q},\left\{a^{0}\right\}\right)
$$

is a set of $\left\{a^{0}\right\}$-relations which defines an $A_{2}$-structure on $\bar{M}_{1}$. By definition

$$
M_{0}=A \text { and } M_{1}=\bar{M}_{1} \text {. }
$$

Suppose also that $\gamma$ is the natural map

$$
\gamma:\left[\bar{M}_{1}, \bar{M}_{1}\right] \rightarrow\left[M_{1}, M_{1}\right]_{A} .
$$


Then since $A$ is contractible ( $B$ being so), we can easily check that the natural map

$$
\bar{M}_{1} \times \bar{M}_{1} \rightarrow M_{1} \times{ }_{A} M_{1}
$$

is a homotopy equivalence. This implies that $\gamma$ is a homotopy equivalence by comparing the homology groups. Hence

$$
\tilde{M}_{2}=\left[M_{1}, M_{1}\right]_{A} \cup_{\gamma} \bar{M}_{2},
$$

where $\bar{M}_{2}$ is attached to $\left[M_{1}, M_{1}\right]_{A}$ by $\gamma$, is of the homotopy type of $M_{2}$, and the two-sided $A$-complex

$$
M_{2}=\tilde{M}_{2} \cup A \times \tilde{M}_{2} \times A,
$$

where $A \times \tilde{M}_{2} \times A$ is attached to $\tilde{M}_{2}$ by the map

$$
A \times\left[M_{1}, M_{1}\right]_{A} \times A \cup \tilde{M}_{2} \rightarrow \tilde{M}_{2},
$$

is of the homotopy type of $M_{1}$. To find an invariant two-sided deformation retraction of $M_{2}$ onto $M_{1}$, apply Lemma (7.1) of $\S 7$. Thus, with $\mu_{p, q}$ being the natural maps,

$$
\mathscr{M}_{2}\left(M_{1}\right)=\left(M_{0}, M_{1}, M_{2} ; \mu_{p, q}, A\right)
$$

is a set of $A$-relations which defines a $Q_{2}$-structure on $M_{1}$.

Observe that the first part of the proof did not use the fact that $B$ is contractible. In fact, the same argument can show that any set of $A$-relations on a two-sided $A$-complex $M_{1}$ defines a set of $A^{\prime}$-relations on $M_{1} . A^{\prime}$ being any RPT-subcomplex of $A_{1}$. The two sets are related but do not define the same RPT-complex.

6. Fibrations of the Hopf type defined by a $Q_{n}$-structure. One of the attractive features of the Hopf fibrations defined by an $H$-structure on a space is that they enable one to reduce the property of the existence of an $H$-structure, which is essentially an algebraic property, to the existence of fibrations and projective spaces defined by them (see e.g., [9]). In fact, this has been one of the most productive tools in the study of $H$-spaces. We shall see that the $A$-relations defining a $Q_{n}$-structure provide us with fibrations on the Hopf type. First we need introduce the concept of smashed product and join in the category of $A$-spaces. Suppose $M_{1}$ is a twosided $A$-complex, and let ${ }^{2} M$ be the RPT-complex generated by $M_{1}$ modulo the set of $A$-relations

$$
\mathscr{M}_{2}\left(M_{1}\right)=\left(M_{0}, M_{1},\left[M_{1}, M_{1}\right]_{A} ; \mu_{p, q}, A\right)
$$

where $\mu_{p, q}$ are the natural maps. Consider

$$
[]_{A}^{n}\left(M_{1}\right)=\left[M_{1}, \cdots, M_{1}\right]_{A},
$$

the subcomplex of ${ }^{2} M$ of index $n$. Then []$_{A}^{n}\left(M_{1}\right)$ is a two-sided $A$-complex 
which includes []$_{A}^{n-1}\left(M_{1}\right)$ as a two-sided $A$-subcomplex. By definition, the $n$-fold smashed product of $M_{1} / / A$ relative to $A$ is

$$
(\#)_{A}^{n}\left(M_{1} / / A\right)=\left([]_{A}^{n}\left(M_{1}\right) / / A\right) /\left([]_{A}^{n-1}\left(M_{1}\right) / / A\right),
$$

the special complex obtained from []$_{A}^{n}\left(M_{1} / / A\right)$ by identifying the subcomplex []$_{A}^{n-1}\left(M_{1} / / A\right)$ with the basepoint. Note that if $A=\left\{a^{0}\right\}$, where $a^{0}$ is a single 0 -cell, then

$$
(\#)_{A}^{n}\left(M_{1} / / A\right)=(\#)^{n}\left(M_{1}\right)
$$

the usual smashed product, since in this case $M_{1} / / A=M_{1}$. By definition, the $n$-fold join of $M_{1} / / A$ relative to $A$ is

$$
\left({ }^{*}\right)_{A}^{n}\left(M_{1} / / A\right)=S^{n-1} \#(\#)_{A}^{n}\left(M_{1} / / A\right),
$$

where $S^{n-1} \#(\#)_{A}^{n}\left(M_{1} / / A\right)$ is the usual smashed product of the $(n-1)$-sphere and $(\#)_{A}^{n}\left(M_{1} / / A\right)$. Again observe that if $A=\left\{a^{0}\right\}$, then the join relative to $\left\{a^{0}\right\}$ is just the usual join.

Suppose that $M_{1}$ is a two-sided $A$-complex, and let

$$
\mathscr{M}_{n}\left(M_{1}\right)=\left(M_{0}, \cdots, M_{n} ; \mu_{p, q}, A\right)
$$

be a set of relations which defines a $Q_{n}$-structure on $M_{1}$ (see Definition (5.4) above). Denote by ${ }^{k} M$ the RPT-complex generated by $M_{1}$ modulo the subset $\mathscr{M}_{k}\left(M_{1}\right), k=2, \cdots, n$, and by $B_{k}$, the basespace of the universal quasi-fibration of ${ }^{k} M$ (see $\S 4$ ). We have then the following theorem.

THEOREM (6.3). There is a commutative diagram,

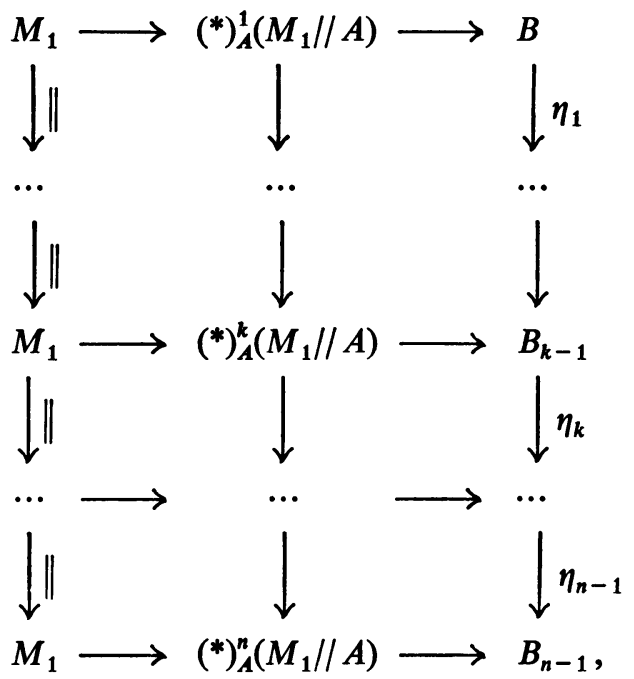

where the rows are fiber sequences, the maps $\eta_{k}$ are those induced by the imbed- 
dings ${ }^{k-1} M \rightarrow{ }^{k} M$, and $\left({ }^{*}\right)_{A}^{k}\left(M_{1} / / A\right)$ is the $k$-fold join of $M_{1} / / A$ relative to $A$. Moreover, $M_{1}$ is contractible in $\left({ }^{*}\right)_{A}^{k}\left(M_{1}^{k} / / A\right)$, for $k \geqq 2$, and $\left({ }^{*}\right)_{A}^{k}\left(M_{1} / / A\right)$ is contractible in $\left(^{*}\right)_{A}^{k+1}\left(M_{1} / / A\right)$, for $k \geqq 1$.

The proof will be given in $\S 8$ below. Note that if $A=\left\{a^{0}\right\}$, then the preceding fibrations are the usual Hopf fibrations associated with an $A_{n}$-structure [11].

Suppose now that Condition (5.5) is satisfied, and assume

$$
\mathscr{M}_{n}\left(M_{1}\right)=\left(M_{0}, \cdots, M_{n} ; \mu_{p, q}, A\right)
$$

is a set of $A$-relations which defines a $Q_{n}$-structure on $M_{1}$. Then Theorem (6.3) implies that there is a ladder of fiber sequences

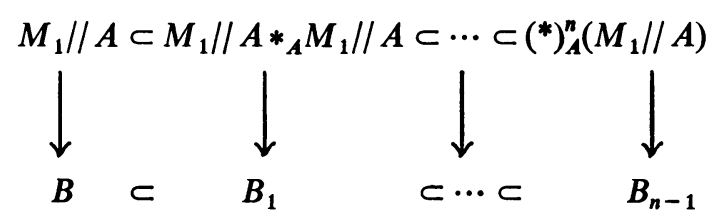

with fiber $M_{1}$. Observe that, from the second stage on, $M_{1}$ is contractible in the total space $\left({ }^{*}\right)_{A}^{k}\left(M_{1} / / A\right)$, while $\left({ }^{*}\right)_{A}^{k}\left(M_{1} / / A\right)$ is always contractible in $\left({ }^{*}\right)_{A}^{k+1}\left(M_{1} / / A\right)$. This implies that

$$
B_{k} \sim B_{k-1} \cup T\left(\left(^{*}\right)_{A}^{k}\left(M_{1} / / A\right)\right) \cup \text { other cells, }
$$

where $T\left(\left(^{*}\right)_{A}^{k}\left(M_{1} / / A\right)\right)$ is the cone on $\left({ }^{*}\right)_{A}^{k}\left(M_{1} / / A\right)$. The fact that $\phi$ (see (5.5)) is a right invariant map which is a homotopy equivalence implies that the induced map

$$
\bar{\phi}: U / / A=F \rightarrow M_{1} / / A
$$

is also a homotopy equivalence. But what can we say about $\left(^{*}\right)_{A}^{k}\left(M_{1} / / A\right)$, for $k \geqq 2$ ? A partial answer is given by the following proposition.

Proposition (6.5). Suppose $F$ is a suspension. Then there is a natural homotopy equivalence

$$
w:\left({ }^{*}\right)_{A}^{k}\left(M_{1} / / A\right) \rightarrow\left({ }^{*}\right)^{k}(F), k \geqq 1 .
$$

Proof. Suppose that

$$
F=S \# F,
$$

where $F$ is a special complex. Since $F$ is a subcomplex of $B, F_{\infty}$ is an RPT-subcomplex of $A$, the RPT-complex representing $\Omega(B)$ as in (5.1) above. This means that

$$
U=E\left(F_{\infty}\right) \times \bar{F}_{\infty} A,
$$

where $E\left(F_{\infty}\right)$ is the universal quasi-fibration of $F_{\infty}$ (see Corollary (4.5)), and 


$$
E\left(F_{\infty}\right)=F_{\infty} \cup T F \times F_{\infty}
$$

(see proof of Corollary (4.5)). Hence

$$
\bar{U}=A \cup T F
$$

where the cone $T F$ is attached by the imbedding on the base is a subcomplex of $U$. Note that

$$
\bar{U} / A=U / / A,
$$

and hence $\bar{U} / A$ and $M_{1} / / A$ are of the same homotopy type. To finish the proof, note that the imbedding of $\bar{U}$ induces a map,

$$
t:(\bar{U} / A) \#(\bar{U} / A) \rightarrow\left(M_{1} / / A\right) \#_{A}\left(M_{1} / / A\right) .
$$

Since $U$ is right invariant homotopy equivalent to $M_{1}$ (see Remark (7.2) of $\S 7$ ), it follows that $(U / / A) \#_{A}\left(M_{1} / / A\right)$ (defined by identifying the image of $U \times A \cup A \times M_{1}$ in $\left(U \times{ }_{A} M_{1}\right) / / A$ with the basepoint $)$ is homotopy-equivalent to $\left(M_{1} / / A\right) \#_{A}\left(M_{1} / / A\right)$. But

$$
(U / / A) \#_{A}\left(M_{1} / / A\right)=(\bar{U} / A) \#\left(M_{1} / / A\right),
$$

since $U=\bar{U} \cup \bar{U} \times A$, the attaching map being the multiplication

$$
A \times A \rightarrow A \subset \bar{U} .
$$

This is enough to prove that $t$ is a homotopy equivalence and, therefore, the proposition when $k=2$. The general case follows similarly by induction.

Rewriting (6.4), we get the following

$$
B\left({ }^{k} M\right) \sim B\left({ }^{k-1} M\right) \cup T\left(\left({ }^{*}\right)_{A}^{k} F\right) \cup \text { other cells, }
$$

when $F$ is a suspension. How are the "other cells" related to $F$ and $B$ ? This turns out to be a tractable problem. A complete description of how they are formed out of $F$ and $B$ and how they are attached to $B\left({ }^{k-1} M\right) \cup T\left(\left({ }^{*}\right)_{A} F\right)$, as well as some applications of these results, will be given in a future article. Here we shall note only that, if $B$ is contractible, then these extra cells do not change the homotopy type of $B\left({ }^{k-1} M\right) \cup T\left(\left({ }^{*}\right)_{A}^{k} F\right)$. To see this, apply Theorem (5.9) of $\S 5$ to replace the given set of $A$-relations,

$$
\mathscr{M}_{n}\left(M_{1}\right)=\left(M_{0}, \cdots, M_{n} ; \mu_{p, q}, A\right),
$$

defining the $Q_{n}$-structure on $M_{1}=\Omega(B, F)$ by a set

$$
\mathscr{M}_{n}\left(\bar{M}_{1}\right)=\left(\bar{M}_{0}, \cdots, \bar{M}_{n} ; \mu_{p, q},\left\{a^{0}\right\}\right)
$$

of relations which defines an $A_{n}$-structure on $\bar{M}_{1}=M_{1}$. We also have a homomorphism

$$
f_{k}:{ }^{k} \bar{M} \rightarrow{ }^{k} M, \quad k=1, \cdots, n,
$$


of the RPT-complex ${ }^{k} \bar{M}$ generated by $\bar{M}_{1}$ modulo $\mathscr{M}_{k}\left(\bar{M}_{1}\right)$ into the RPT-complex ${ }^{k} M$ generated by $M_{1}$ modulo $\bar{M}_{k}\left(M_{1}\right)$. Now we note that $f_{k}$ induces a map

$$
\tilde{f}_{k}: B\left({ }^{k} \bar{M}\right) \rightarrow B\left({ }^{k} M\right)
$$

and that Theorem (6.3) implies that $\tilde{f}_{k}$ is a homotopy equivalence, which is enough to prove the assertion.

Thus, if $B$ is contractible, $B\left({ }^{n} M\right)$ is the projective space of $F$ corresponding to the $A_{n}$-structure which is defined by the given $Q_{n}$-structure, and conversely.

It is well known that $F$ is an $H$-space if, and only if, $F_{\infty}=\Omega S \# F$ retracts onto $F$ (e.g., see [4]). This result was extended to $A_{n}$-structures in [3]: $F$ admits an $A_{n}$-structure if and only if, $\Omega\left(P_{n}(F)\right)$ can be retracted onto $F, P_{n}(F)$ being the $n$-projective space of $F$. The following theorem gives a similar result for $Q_{n}$ structures.

Theorem (6.5). Suppose $M_{1}$ is a two-sided A-complex, $A$ being an RPTcomplex, and assume that $M_{1}$ admits a $Q_{n}$-structure defined by the set of A-relations

$$
\mathscr{M}_{n}\left(M_{1}\right)=\left(M_{0}, \cdots, M_{n} ; \mu_{p, q}, A\right) .
$$

Then this $Q_{n}$-structure can be extended to a $Q_{n+1}$-structure if there is a twosided invariant retraction,

$$
r_{n+1}:{ }^{n} M \rightarrow M_{n},
$$

where ${ }^{n} M$ is the RPT-complex generated by $M_{1}$ modulo $\mathscr{M}_{n}\left(M_{1}\right)$ and $M_{n}$ is the subcomplex of ${ }^{n} M$ of index $n$.

Conversely, if the given $Q_{n}$-structure can be extended to a $Q_{n+1}$-structure, then there is an invariant right retraction of ${ }^{n} M$ onto $M_{n}$.

The fact that a $Q_{n}$-structure can be extended to a $Q_{n+1}$-structure if $r_{n+1}$ exists can be proved in the same way as Theorem (4.6) of [3], the only modification being to replace "complex" by " $A$-complex" and "map" by "invariant map." To prove the converse, apply Theorem (6.3) to prove that

$$
K_{\infty} \times M_{n} \text { and }{ }^{n} M \text { are of the same homotopy type, }
$$

( $K$ being a special complex such that $S \not K=(\#)_{A}^{n+1}\left(M_{1} / / A\right)$ ), and then apply Remark (7.2) of $\S 7$ to the mapping cylinder of the homotopy equivalence to get the right invariant retraction. (Consider $K_{\infty} \times M_{n}$ as a right $A$-complex by making $A$ act on $M_{n}$.)

7. Proof of Theorem (5.3). In general outline the proof resembles that of Theorem (4.5) of [3], but some of the details are different. Suppose

$$
F \stackrel{i}{\longrightarrow} B \stackrel{f}{\longrightarrow} Y
$$


is a fiber sequence of special complexes, where $B$ and $F$ are simply connected and $F$ is a subcomplex of $B$. Assume also that $B$ is a suncomplex of $Y$. As in $\S 5$, let

$$
g: A \rightarrow \Omega(B)
$$

be a homomorphism of the RPT-complex $A$ into $\Omega(B)$, which is also a homotopy equivalence, and

$$
g^{\prime}: U \rightarrow \Omega(B, F),
$$

the map of an $A$-space into an $\Omega(B)$-space, which is a homotopy equivalence (see (5.1) and (5.2)). Now, according to [3], there is an RPT-complex $M$, possibly of infinite rank, which contains $A$ as a sub- $H$-complex, and a homomorphism,

$$
\eta: M \rightarrow \Omega(Y),
$$

which extends $g$ and is a homotopy equivalence. Clearly $M$ is a two-sided $A$-complex.

By definition, let

$$
M_{0}=A \text { and } M_{1}=M .
$$

Next, let

$$
\tilde{M}_{2}=\left[M_{1}, M_{1}\right]_{A} .
$$

Then the mapping

$$
\tilde{\eta}_{2}: \tilde{M}_{2} \rightarrow M_{1}
$$

which sends the point $\left[x_{1}, x_{2}\right]$ to $\gamma\left(x_{1}, x_{2}\right)$, where $x_{1}, x_{2} \in \tilde{M}_{2}$ and $\gamma$, the multiplication in $M_{1}$, is a two sided invariant retraction of $\tilde{M}_{2}$ onto $M_{1}$. By definition, let

$$
M_{2}=\widetilde{M}_{2} \cup_{\beta_{2}} \widetilde{M}_{2} \times I
$$

where $I$ is the unit interval $[0,1]$ and the attaching map $\beta_{2}$ is defined by the equations

$$
\begin{aligned}
& \beta_{2}(x, 0)=x, \beta_{2}(x, 1)=\tilde{\eta}_{2}(x), \text { for } x \in \tilde{M}_{2}, \text { and } \\
& \beta_{2}(x, t)=x, \text { for }(x, t) \in M_{1} \times I .
\end{aligned}
$$

Now, if we make $A$ act on $M_{2}$ according to the rule

$$
\begin{gathered}
a(x, t)=(a x, t) \text { and } \\
(x, t) a=(x a, t), .
\end{gathered}
$$

for all $(x, t) \in M_{2}$ and $a \in A$, then $M_{2}$ becomes a two-sided $A$-complex, and the map

$$
{ }^{2} h_{s}: M_{2} \rightarrow M_{2},
$$


which sends the point $(x, t)$ to $(x,(1-s) t+s)$, is a two-sided invariant deformation retraction of $M_{2}$ onto $M_{1}$. Hence the set

$$
\mathscr{M}_{2}\left(M_{1}\right)=\left(M_{0}, M_{1}, M_{2} ; \mu_{p, q}, A\right),
$$

where the complexes $M_{i}$ are defined above and $\mu_{p, q}$ are the natural maps, is a set of $A$-relations on $M_{1}$, which defines a $Q_{2}$-structure on $M_{1}$ as desired (see Definition (5.4)).

Proceed by induction. Suppose that we have been able to define a set of $A$-relations

$$
\mathscr{M}_{m-1}\left(M_{1}\right)=\left(M_{0}, \cdots, M_{m-1} ; \mu_{p, q}, A\right)
$$

and a two-sided invariant mapping,

(i) ${ }^{j} h: M_{j} \times I \rightarrow M_{j}, j=2, \cdots, m-1$,

which is a deformation retraction of $M_{j}$ onto $M_{j-1}$, and a two-sided invariant retraction

(ii) $\eta_{j}: M_{j} \rightarrow M_{1}, j=2, \cdots, m-1$

such that

$$
\gamma\left(\eta_{p} \times \eta_{q}\right)=\eta_{p+q} \mu_{p, q}, \quad p, q \geqq 0 \text { and } p+q>m-1,
$$

$\gamma$ being the multiplication in $M=M_{1}$. We wish to do the same thing for the case $m$. Consider, therefore,

$$
\tilde{M}_{m}=\bigcup\left[M_{p}, M_{q}\right]_{A}, \text { with } p+q=m,
$$

the subcomplex of index $m$ of the RPT-complex ${ }^{m-1} M$ generated by $M_{1}$ modulo $\mathscr{M}_{m-1}\left(M_{1}\right)$. Since the mappings $\eta_{j}$ and $\gamma$ are two-sided invariant and associative, the maps $\eta_{j}$ induce together a two-sided invariant retraction,

such that

$$
\tilde{\eta}_{m}: \tilde{M}_{m} \rightarrow M_{1} \text {, }
$$

$$
\tilde{\eta}_{m} \mid M_{k}=\eta_{k}, \text { with } 1 \leqq k \leqq m-1
$$

By definition, let

$$
\bar{M}_{m}=\tilde{M}_{m} \cup \tilde{M}_{m}+I,
$$

where $I$ is the unit interval $[0,1]$ and the attaching map $\beta_{m}$ is defined by the equations

$$
\begin{aligned}
& \beta_{m}(x, 0)=x, \beta_{m}(x, 1)=\eta_{m}(x), \text { for } x \in \tilde{M}_{m}, \text { and } \\
& \beta_{m}(x, t)=x, \text { for }(x, t) \in M_{1} \times I .
\end{aligned}
$$

Now, if we make $A$ act on $\bar{M}_{m}$ by the rule

$$
\begin{aligned}
& a(x, t)=(a x, t) \text { and } \\
& (x, t) a=(x a, t),
\end{aligned}
$$


for all $(x, t) \in \bar{M}_{m}$, then $\bar{M}_{m}$ becomes a two-sided $A$-complex. Define

$$
\eta_{m}: \bar{M}_{m} \rightarrow M_{1}
$$

to be the map that takes $(x, t)$ to $\tilde{\eta}_{m}(x)$. Clearly the set

$$
\mathscr{M}_{m}\left(\bar{M}_{1}\right)=\left(\bar{M}_{0}, \cdots, \bar{M}_{m} ; \bar{\mu}_{r, q}, A\right) \text {, }
$$

where $\bar{M}_{k}=M_{k}$, for $k<m$, and $\bar{\mu}_{p, q}$ are the natural maps, is a set of $A$-relations which satisfies (ii) of the induction hypothesis and which extends $\mathscr{M}_{m-1}\left(M_{1}\right)$. Thus, to finish the induction step, we need find a two-sided invariant map

$$
{ }^{m} h: \bar{M}_{m} \times I \rightarrow \bar{M}_{m}
$$

which deforms $\bar{M}_{m}$ onto $\bar{M}_{m-1}$.

Note that the imbedding

$$
\gamma: \bar{M}_{m-1} \rightarrow \bar{M}_{m}
$$

is a homotopy equivalence, since each of the complexes is of the homotopy type of $\bar{M}_{1}=M_{1}$. Hence we need prove the following lemma only.

Lemma (7.1). Suppose $N$ and $N^{\prime}$ are two-sided A-complexes, and assume that there is an imbedding

$$
\gamma: N \rightarrow N^{\prime}
$$

which is a homotopy equivalence. Then there is a two-sided invariant deformation retraction

$$
\phi_{t}: N^{\prime} \rightarrow N^{\prime}
$$

of $N^{\prime}$ onto $N$.

Proof. According to $\S 1$, there is an ascending sequence of two-sided $A$-complexes,

$$
N=N_{0} \subset \cdots \subset N_{k} \subset \cdots \subset N^{\prime},
$$

such that $N^{\prime}=\bigcup_{k} N_{k}$ and, for each $k \geqq 1$,

$$
N_{k}=\tilde{N}_{k} \cup A \times \tilde{N_{k}} \times A,
$$

where $\tilde{N}_{k}$ is the subcomplex

$$
\tilde{N}_{k}=N_{k-1} \cup_{f_{k}} D^{n_{k}}
$$

( $D^{n_{k}}$ being the $n_{k}$-disc attached to $N_{k-1}$ by some map $f_{k}$ ) and the complex $A \times \tilde{N}_{k} \times A$ is attached to $\tilde{N}_{k}$ by the map

$$
A \times N_{k-1} \times A \cup \tilde{N}_{k} \rightarrow \tilde{N}_{k},
$$

which is induced by the multiplication by $A$. We shall construct the deformation 
$\phi_{t}$ by induction on the two-sided $A$-subcomplexes $N_{k}$. Now, since the imbedding $\gamma$ of $N$ into $N^{\prime}$ is a homotopy equivalence, the attaching map

$$
f_{1}:\left(D^{n_{1}}, S^{n_{1}-1}\right) \rightarrow\left(N^{\prime}, N\right)
$$

can be deformed rel $S^{n_{1}-1}$, into a map of $D^{n_{1}}$ into $N$. This deformation yields a deformation retraction,

$$
\left(\tilde{\phi}_{t}\right)_{1}: \tilde{N}_{1} \rightarrow N^{\prime},
$$

of $\tilde{N}_{1}$ onto $N$ which is extendable uniquely to a two-sided invariant deformation retraction,

$$
\left(\phi_{t}\right)_{1}: N_{1} \rightarrow N^{\prime},
$$

of $N_{1}$ onto $N$. Suppose that, by induction, we have been able to find a two-sided $A$-deformation retraction,

$$
\left(\phi_{t}\right)_{m-1}: N_{m} \rightarrow N^{\prime},
$$

of $N_{m}$ onto $N$. Consider the characteristic map

$$
f_{:}:\left(D^{n_{m}}, S^{n_{m}-1}\right) \rightarrow\left(\tilde{N}_{m}, N_{m-1}\right) .
$$

Note that we have a deformation of $S^{n_{m}-1}$ into $N$ defined by $\left(\phi_{t}\right)_{m-1}$. By the homotopy extension property of the pair $\left(D^{n_{m}}, S^{n_{m}-1}\right)$, we get a deformation of $f_{m}$ into a map

$$
f_{m}^{\prime}:\left(D^{n_{m}}, S^{n_{m}-1}\right) \rightarrow\left(N^{\prime}, N\right) .
$$

But and $N^{\prime}$ are of the same homotopy type. Thus we get a deformation,

$$
\left(\tilde{\phi}_{t}\right)_{m}: \tilde{N}_{m} \rightarrow N^{\prime},
$$

of $\tilde{N}_{m}$ onto $N$ which extends $\left(\phi_{t}\right)_{m-1}$. Then $\left(\tilde{\phi}_{t}\right)_{m}$ can be extended uniquely to a two-sided invariant deformation retraction,

$$
\left(\phi_{t}\right)_{m}: N_{m} \rightarrow N
$$

of $N_{m}$ onto $N$ as desired.

REMARK (7.2). A similar argument proves that, if $N$ and $N^{\prime}$ are right (left) $A$-complexes and $\gamma$ is a right (left) $A$-imbedding, then one can find a right (left) invariant deformation retraction $\phi_{t}$ of $N^{\prime}$ onto $N$.

8. Proof of Theorem (6.3). Suppose that $M_{1}$ is a two-sided $A$-complex which admits a $Q_{n}$-structure defined by the set of $A$-relations

$$
\mathscr{M}_{n}\left(M_{1}\right)=\left(M_{0}, \cdots, M_{n} ; \mu_{p, q}, A\right) \text {. }
$$

Denote the subset of $\mathscr{M}_{n}\left(M_{1}\right)$ in $k-1$ variables by

$$
\mathscr{M}_{k-1}\left(\bar{M}_{1}\right)=\left(\bar{M}_{0}, \cdots, \bar{M}_{k-1} ; \bar{\mu}_{p, q}, A\right) \text {. }
$$


Suppose ${ }^{k-1} M$ is the RPT-complex generated by $\bar{M}_{1}$ modulo $\mathscr{M}_{k-1}\left(\bar{M}_{1}\right)$, and consider the complex

$$
{ }^{k} N={ }^{k-1} M \cup M_{k} \times{ }_{A}^{k-1} M,
$$

where the complex $M_{k} \times{ }_{A}{ }^{k-1} M$ is attached to ${ }^{k-1} M$ by the map

$$
\bar{M}_{k} \times{ }^{k-1} M \rightarrow{ }^{k-1} M
$$

which is induced by the multiplication in ${ }^{k-1} M$. (Here $\bar{M}_{k}={ }^{k-1} M \cap M_{k}$, the subcomplex of ${ }^{k-1} M$ of index $k$.) Now let ${ }^{k-1} M$ act on ${ }^{k} N$ on the right, thereby making ${ }^{k} N$ a right ${ }^{k-1} M$-complex. Hence, by Theorem (2.2) of $\S 2$, the sequence

$$
{ }^{k-1} M \rightarrow{ }^{k} N \rightarrow{ }^{k} N / /{ }^{k-1} M,
$$

is a quasi-fibration and, hence, a fiber sequence. The fibrations of the Hopf type are induced in the following way by (8.2). Denote by $B_{k-1}$ the base-space of the universal quasi-fibration of ${ }^{k-1} M$ (see (4.5) of $\S 4$ ). Then the sequence

$$
{ }^{k} N \rightarrow{ }^{k} N / /{ }^{k-1} M \stackrel{q_{k}}{\longrightarrow} B_{k-1}
$$

is a fiber sequence, where $q_{k}$ is the classifying map of (8.2) $)_{k}$. Observe that (8.3) maps into $(8.3)_{k+1}$ in a natural fashion. Thus we get the commutative ladder,

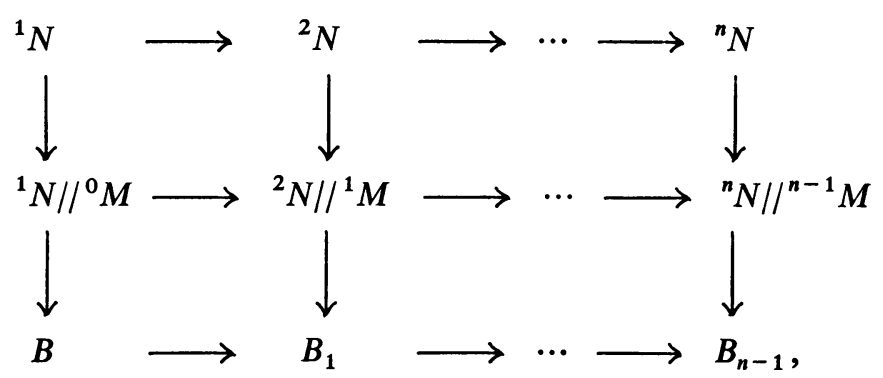

where ${ }^{0} M=A$, the vertical sequences are fiber sequences, and the maps of the lowest sequence are those induced by the homomorphisms ${ }^{k} M \rightarrow^{k+1} M$. Thus, to finish the proof of the theorem, we need prove the following two lemmas only.

LEMMA (8.4). The natural imbedding

$$
M_{k} \rightarrow{ }^{k} N \text {, }
$$

where $M_{k}$ is the $k$ th complex of $\mathscr{M}_{k}\left(M_{1}\right)$ and ${ }^{k} N$ is the complex defined above, is a homotopy equivalence.

LemMa (8.5). The complexes

$$
{ }^{k} N / /{ }^{k-1} M \text { and }\left({ }^{*}\right)_{A}^{k}\left(M_{1} / / A\right)
$$

are of the same homotopy type. 
Proof of Lemma (8.4). Recall that the RPT-complex ${ }^{k-1} M$ is filtered by an ascending sequence of two-sided $A$-complexes

$$
\left({ }^{k-1} M\right)_{r}=\bigcup_{p=1}^{k-1}\left[\bar{M}_{p}, \bar{M}_{r-p}\right]_{A}
$$

such that

$$
\left[\bar{M}_{p}, \bar{M}_{r-p}\right]_{A} \cap \bigcup_{q<p}\left[\bar{M}_{q}, \bar{M}_{r-q}\right]_{A}=\bigcup_{q<p}\left[\bar{M}_{p-q}, \bar{M}_{q}, \bar{M}_{r-p}\right]_{A}
$$

where $\bar{M}_{i}$ is the subcomplex of ${ }^{k-1} M$ of index $i, i \geqq 1$ (see Corollary (3.7) of $\S 3$ ). It can be easily checked that this implies that

$$
\left({ }^{k-1} M\right)_{r}=\left[\bar{M}_{k}, \bar{M}_{r-k}\right]_{A} \cup \bigcup_{p=1}^{k-2}\left[\bar{M}_{k-p}, \bar{M}_{r-k+p}\right]_{A},
$$

where

$$
\left[\bar{M}_{k-q}, \bar{M}_{r-k+q}\right]_{A} \cap \bigcup_{p=0}^{q-1}\left[\bar{M}_{k-p}, \bar{M}_{r-k+p}\right]_{A}=\bigcup_{p=0}^{q-1}\left[\bar{M}_{k-q}, \bar{M}_{q-p}, \bar{M}_{r-k+p}\right]_{A} .
$$

Therefore we can filter the complex ${ }^{k} N$ by an ascending sequence of two-sided $A$-subcomplexes,

$$
\left({ }^{k} N\right)_{r}=\left[M_{k}, \bar{M}_{r-k}\right]_{A} \cup \bigcup_{p=1}^{k-2}\left[\bar{M}_{k-p}, \bar{M}_{r-k+p}\right]_{A}, \quad r \geqq k,
$$

such that

$$
\begin{aligned}
\left(\left[M_{k}, \bar{M}_{r-k}\right]_{A}\right. & \left.\cup \bigcup_{p=1}^{q-1}\left[\bar{M}_{k-p}, \bar{M}_{r-k+p}\right]_{A}\right) \cap\left[\bar{M}_{k-q}, \bar{M}_{r-k-q}\right]_{A} \\
& =\bigcup_{p=0}^{q-1}\left[\bar{M}_{k-q}, \bar{M}_{q-p}, \bar{M}_{r-k+p}\right]_{A}
\end{aligned}
$$

where $\bar{M}_{i}$ is the subcomplex of ${ }^{k-1} M$ of index $i$. Observe now that $\bar{M}_{k-1}$ is a two-sided $A$-deformation retract of $M_{k}$, and, for $p \geqq 1, \bar{M}_{k-p-1}$ is a two-sided $A$-deformation retract of $\bar{M}_{k-p}$. Hence $\left({ }^{k} N\right)_{r}$ is made up of complexes each of which is of the homotopy type of $\left({ }^{k} N\right)_{r-1}$. One can show inductively, using the Mayer-Vietoris sequences of the appropriate proper triads which correspond to the decomposition of $\left({ }^{k} N\right)_{r}$ given above, that in constructing $\left({ }^{k} N\right)_{r-1}$ one attaches two complexes of the homotopy type of $\left({ }^{k} N\right)_{r-1}$ along a subcomplex of the homotopy type of $\left({ }^{k} N\right)_{r-1}$. This establishes the lemma.

Proof of Lemma (8.5). If $Q$ is a subcomplex of the special complex $P$, then denote by $P / Q$ the special complex obtained from $P$ by identifying $Q$ with the base point. Observe that

$$
\left(M_{k} / / A\right) /\left(\bar{M}_{k} / / A\right)={ }^{k} N / /{ }^{k-1} M .
$$


Hence it suffices to prove that

$$
\left(M_{k} / / A\right) /\left(\bar{M}_{k} / / A\right) \text { and } S^{k-1} \#(\#)_{A}^{k}\left(\bar{M}_{1} / / A\right)
$$

are of the same homotopy type.

Proceed by induction. If $k=1$, then the assertion is trivially true. Suppose we have been able to prove $(8.6)_{m-1}$. To prove $(8.6)_{m}$, write

$$
[p, q, r]=\left(\left[\bar{M}_{p}, \bar{M}_{q}, \bar{M}_{r}\right]_{A}\right) / / A ;
$$

similarly, write $[p]$ for $\left(\bar{M}_{p}\right) / / A$, and $[p, q]$ for $\left(\left[\bar{M}_{p}, \bar{M}_{q}\right]\right) / / A$. Let us also put

$$
[p] \#_{A}[q] \#_{A}[r]=[p, q, r] /[(p-1, q, r] \cup[p, q-1, r] \cup[p, q, r-1]) .
$$

Observe now that Corollary (3.7) of $\S 3$ implies that

where

$$
[m] /[m-1]=\bigcup_{p=1}^{m-1}[m-p] \#_{A}[p]
$$

$$
[m-q] \#_{\boldsymbol{A}}[q] \cap \bigcup_{p<q}[m-p] \#_{\boldsymbol{A}}[p]=\bigcup_{p<q}[m-q] \#_{\boldsymbol{A}}[q-p] \#_{\boldsymbol{A}}[p]
$$

To finish the proof we need the following lemma.

LEMMA $(8.7)_{m}$. The complex

is contractible,

$$
\bigcup_{p=1}^{m-2}[m-p] \#_{A}[p]
$$

Proof. Proceed by induction on $m$. If $m=3$, then the Lemma is true obviously. Suppose $(8.7)_{s-1}$ is true, with $s \leqq m$. To prove $(8.7)_{s}$, argue by induction. Note that $[s-1] \#_{A}[1]$ is contractible, since $M_{s-2}$ is a two-sided $A$-deformation retract of $M_{s-1}$. Suppose that we have been able to show that

$$
\bigcup_{p=1}^{r-1}[s-p] \#_{A}[p], r-1<s-2
$$

is contractible. Observe that

$$
\bigcup_{p=1}^{r}[s-p] \#_{A}[p]=[s-r] \#_{A}[r] \cup \bigcup_{p=1}^{r-1}[s-p] \#_{A}[p],
$$

and

$$
\bigcup_{p=1}^{r-1}[s-r] \#_{A}[r-p] \#_{A}[p]=[s-r] \#_{A}[r] \cap \bigcup_{p=1}^{r-1}[s-p] \#_{A}[p] .
$$

Next note that $[s-r] \#_{A}[r]$ is contractible, since $M_{r-1}$ is a two-sided $A$-deformation retract of $M_{r}$, for $r=2, \cdots, m \leqq k$. The complexes 


$$
\bigcup_{p=1}^{r-1}[s-p] \#_{A}[p] \text { and } \bigcup_{p=1}^{r-1}[s-r] \#_{A}[r-p] \#_{A}[p]
$$

are also contractible, the first by induction and the second because $M_{r-1}$ is a two-sided deformation retract of $M_{r}$, for $2 \leqq r \leqq m$. Hence

$$
\bigcup_{p=1}^{r}[s-p] \#_{A}[p], \quad r \leqq s-2,
$$

is contractible. This establishes the induction step, and hence, Lemma (8.7).

Now we can go back to the proof of $(8.6)_{m}$. Note that

$$
\bigcup_{p=1}^{m-1}[m-p] \#_{A}[p]=\bigcup_{p=1}^{m-2}[m-p] \#_{A}[p] \cup[1] \#_{A}[m-1],
$$

and

$$
\begin{aligned}
\bigcup_{p=1}^{m-2}[m-p] \#_{A}[p] \cap[1] \#_{A}[m-1] & =\bigcup_{p=1}^{m-2}[1] \#_{A}[m-p-1] \#_{A}[p] \\
& =\bigcup_{q=1}^{m-2}[1] \#_{A}[m-1-q] \#_{A}[q]
\end{aligned}
$$

The induction hypothesis implies that

$$
\begin{aligned}
\bigcup_{q=1}^{m-2}[m-1-q] \#{ }_{A}[q] & =([m-1]) /[m-2] \\
& \sim S^{m-2} \#(\#)_{A}^{m-1}[1] .
\end{aligned}
$$

This proves $(8.6)_{m}$ and, hence, the lemma.

9. Proof of Theorem (5.7). Suppose $B$ is a simply connected special complex and $F$, a connected subcomplex. Assume that $A$ and $U$ are the RPT-complex and right $A$-complex representing $\Omega(B)$ and $\Omega(B, F)$, as in (5.1) and (5.2) of $\S 5$. Recall that Condition (5.5) of $\S 5$ says that there is a two-sided $A$-complex $M_{1}$ and a right invariant map,

$$
\phi: U \rightarrow M_{1},
$$

which is a homotopy equivalence. Suppose that

$$
\mathscr{M}_{n}\left(M_{1}\right)=\left(M_{0}, \cdots, M_{n} ; \mu_{p, q}, A\right)
$$

is a set of $A$-relations which defines a $Q_{n}$-structure on $M_{1}$ (see Definition (5.4)), and let

$$
B \stackrel{\eta_{1}}{\longrightarrow} B_{1} \stackrel{\eta_{2}}{\longrightarrow} \cdots \stackrel{\eta}{\longrightarrow} B_{n}
$$


be the ascending sequence of complexes defined by the sequence of RPT-complexes

$$
A \rightarrow{ }^{1} M \rightarrow \cdots \rightarrow{ }^{n} M,
$$

where ${ }^{k} M$ is the RPT-complex generated by $M_{1}$ modulo $\mathscr{M}_{k}\left(M_{1}\right)$ and $B_{k}$ is the base-space of the universal quasi-fibration of ${ }^{k} M$ (see $\S 4$ ). As in $\S 5$, let

$$
F_{k}=\left\{\alpha \in \tilde{P}\left(B_{k}\right) \mid \alpha(0) \in B\right\},
$$

where $\widetilde{P}\left(B_{k}\right)$ is the space of Moore paths ending at the 0 -cell $b^{0}$. Observe that $\Omega\left(B_{k}\right)$ acts on the left on $F_{k}$. The first stage of the proof is just the replacement of $F_{k}$ by an equivalent left $A$-complex. Now note that, according to $\S 4$, one can find a left universal quasi-fibration,

$$
p_{k}^{\prime}: E^{\prime}\left({ }^{k} M\right) \rightarrow B_{k},
$$

such that $E^{\prime}\left({ }^{j} M\right)$ is a subcomplex of $E^{\prime}\left({ }^{k} M\right)$, the imbedding being a map of a left ${ }^{j} M$-complex into a left ${ }^{k} M$-complex, $k>j$ ( ${ }^{0} M=A$, by definition). Moreover, there is a homomorphism $u_{k}$ of ${ }^{k} M$ into $\Omega\left(B_{k}\right)$ which extends to a map,

$$
u_{k}^{\prime}: E^{\prime}\left({ }^{k} M\right) \rightarrow \widetilde{P}\left(P_{k}\right),
$$

of a left ${ }^{k} M$-complex into a left $\Omega\left(B_{k}\right)$-space such that

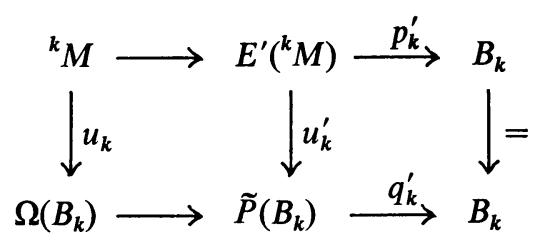

is a commutative diagram. Here $q_{k}^{\prime}$ is the map that sends a path to its initial point. Observe that $u_{k}^{\prime}$ takes ${ }^{k} M \times{ }_{A} E^{\prime}(A)$ into $F_{k}$ and, hence, induces the following commutative diagram:

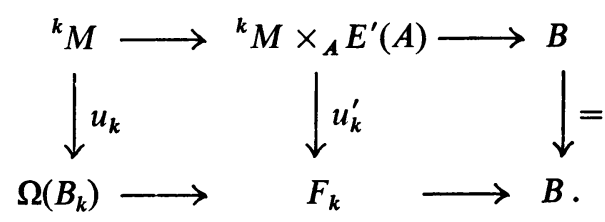

This implies the following assertion.

$$
u_{k}^{\prime} \text { is a homotopy equivalence. }
$$

Thus we can replace $F_{k}$ by the left ${ }^{k} M$-complex ${ }^{k} M \times{ }_{A} E^{\prime}(A)$. Consider now the following diagram (see end of $\S 4$ ), 


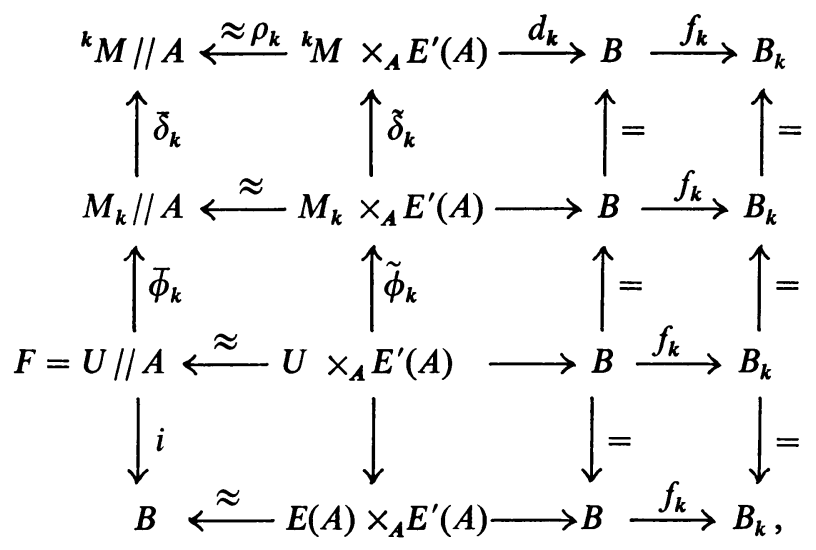

where the horizontal maps to the left of the $B$ column are those induced by the projections on the corresponding factors, while the rest of the horizontal maps and all the vertical maps are those induced by the natural imbeddings, (see end of $\S 4)$. Note that the sequence

$$
{ }^{k} M \times{ }_{A} E^{\prime}(A) \stackrel{d_{k}}{\longrightarrow} B \stackrel{f_{k}}{\longrightarrow} B_{k}
$$

is a fiber sequence which is equivalent to the fiber sequence

$$
\Omega\left(B_{k}, B\right)=F_{k} \stackrel{i_{k}}{\longrightarrow} B \longrightarrow B_{k},
$$

$i_{k}$ being the map that sends a path to its initial point. Observe also that the horizontal maps pointing to the left are all quasi-fibrations with contractible fiber $E^{\prime}(A)$ and hence are homotopy equivalences. Let $\rho_{k}^{-1}$ be a homotopy-inverse for $\rho_{k}$, and set, by definition,

$$
\gamma_{k}=\rho_{k}^{-1} \bar{\delta}_{k} \Phi_{k}
$$

Then $\gamma_{k}$ takes $F=U / / A$ into $F_{k}={ }^{k} M \times{ }_{A} E^{\prime}(A)$, and

$$
\lambda_{k} \gamma_{k} \sim \gamma_{k+1} \text { and } g i=d_{k} \gamma_{k}=i_{k} \gamma_{k}
$$

(note that $d_{k}$ and $i_{k}$ become the same when ${ }^{k} M \times{ }_{A} E^{\prime}(A)$ and $F_{k}$ are identified), where $g$ is a homotopy equivalence of $B$ and $\lambda_{k}$ is the imbedding of ${ }^{k} M \times{ }_{A} E^{\prime}(A)$ into ${ }^{k+1} M \times{ }_{A} E^{\prime}(A)$ induced by the imbedding of ${ }^{k} M$ into ${ }^{k+1} M$ (or, equivalently, the imbedding of $F_{k}$ into $F_{k+1}$ induced by the imbedding $f_{k}$ of $B$ into $B_{k}$ ).

To finish the proof of the theorem, consider the injection

$$
\delta_{k}: M_{k} \rightarrow{ }^{k} M \text {. }
$$

Denote by $V$ the special complex $S^{k-1} \#(\#)_{A}^{k+1}\left(M_{1} / / A\right)$ (see $\left.\S 6\right)$. Then the adjoint $\bar{p}_{k+1}$ of the fibration

$$
p_{k+1}:\left({ }^{*}\right)_{A}^{k+1}\left(M_{1} / / A\right) \rightarrow B_{k}
$$


of Theorem (6.3) induces a homomorphism

$$
\left(\bar{p}_{k+1}\right)_{\infty}: V_{\infty} \rightarrow{ }^{k} M,
$$

where $V_{\infty}$ is the reduced product complex of James. In the usual fashion, Theorem (6.3) implies that the map

$$
v: V_{\infty} \times M_{k} \rightarrow{ }^{k} M
$$

which takes the point $(x, y)$ to $\left(\bar{p}_{k+1}\right)_{\infty}(x) \delta_{k}(y)$ is a homotopy equivalence. Consider now $V_{\infty} \times M_{k}$ a right $A$-complex by allowing $A$ to act on the right factor. Observe that $v$ becomes a right invariant map, and, hence, by Remark (7.2), there is a right $A$-map,

$$
r:{ }^{k} M \rightarrow V_{\infty} \times M_{k},
$$

such that $v r \sim 1$ and $r v \sim 1$. Define

$$
\tilde{\beta}_{k}:{ }^{k} M \rightarrow M_{k}
$$

to be the composition of $r$ and the projection on the second factor. It is a right map and, hence, induces a map

$$
\beta_{k}:{ }^{k} M / / A \rightarrow M_{k} / / A
$$

such that $\beta_{k} \gamma_{k} \sim 1$.

This finishes the proof of part (a) of the theorem. Part (b) can be established as follows.

If $F \sim M_{1} / / A$ is $(c-1)$-connected, then one can replace in a straight forward fashion $M_{1}$ by a two-sided $A$-complex $\tilde{M}_{1}$ of the same homotopy type and such that the cells of lowest dimension in $M_{1} / / A$ are of dimension $c$. Hence $\left({ }^{*}\right)_{A}^{k+1}\left(M_{1} / / A\right)$ is of the homotopy type of $\left({ }^{*}\right)_{A}^{k+1}\left(\tilde{M}_{1} / / A\right)$ (see Lemma (7.1) of \$7). But the cells of lowest dimension in $\left({ }^{*}\right)_{A}^{k+1}\left(\tilde{M}_{1} / / A\right)$ have dimension $(k+1) c$ $+k$. Hence $\left({ }^{*}\right)_{A}^{k+1}\left(M_{1} / / A\right)$ is $((k+1) c+(k-1))$-connected, and $V$ is $((k+1) c$ $+(k-2))$-connected. Therefore, the injection

$$
\delta_{k}: M_{k} \rightarrow^{k} M
$$

induces an isomorphism

$$
\left.\left(\delta_{k}\right)_{*}: \pi_{j}\left(M_{j}\right) \rightarrow \pi_{j}{ }^{k} M\right)
$$

if $j \leqq(k+1) c+(k-2)$.

\section{REFERENCES}

1. A. Dold and R. Lashof, Principal quasifibrations and fiber homotopy equivalence of bundles, Illinois J. Math 3 (1959), 285-305.

2. A. Dold and R. Thom, Quasifaserungen und unendliche symmetrische Produkte, Ann. of Math. 67 (1958), 239-281. 
3. S. Y. Husseini, Constructions of the reduced product type. II, Topology 3 (1965), 59-79.

4. I. M. James, Reduced product spaces, Ann. of Math. 62 (1955), 170-197.

5. - Multiplication on spheres (II), Trans. Amer. Math. Soc. 84 (1957), 545-558.

6. W. S. Massey, Some problems in algebraic topology and the theory of fiber bundles, Ann. of Math. 62 (1955), 327-359.

7. J. W. Milnor, Constructions of universal bundles. II, Ann. of Math. 67 (1958), 239-281.

8. - On spaces having the homotopy type of a CW-complex, Trans. Amer. Math. Soc. 90 (1959), 272-280.

9. H. Samelson, Groups and spaces of loops, Comment Math. Helv. 28 (1954), 278-287.

10. E. H. Spanier and J. H. C. Whitehead, On fiber spaces in which the fiber is contractible, Comment Math. Helv. 29 (1955), 1-7.

11. J. D. Stasheff, Homotopy-associativity of H-spaces. I, Trans. Amer. Math. Soc. 108 (1963), 275-292.

The UNIVERSITY OF WisCONSIN, MADISON, WISCONSIN 